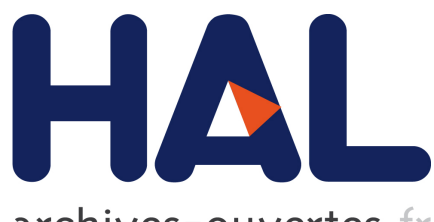

archives-ouvertes

\title{
A 1D Continuum Model for Beams with Pantographic Microstructure: Asymptotic Micro-Macro Identification and Numerical Results
}

Emilio Barchiesi, Francesco Dell'Isola, Marco Laudato, Luca Placidi, Pierre

Seppecher

\section{To cite this version:}

Emilio Barchiesi, Francesco Dell'Isola, Marco Laudato, Luca Placidi, Pierre Seppecher. A 1D Continuum Model for Beams with Pantographic Microstructure: Asymptotic Micro-Macro Identification and Numerical Results. Advances in Mechanics of Microstructured Media and Structures , 87, pp 43-74, 2018, Advanced Structured Materials book series (STRUCTMAT), volume 87. < hal-01817742>

\section{HAL Id: hal-01817742 \\ https://hal.archives-ouvertes.fr/hal-01817742}

Submitted on 18 Jun 2018

HAL is a multi-disciplinary open access archive for the deposit and dissemination of scientific research documents, whether they are published or not. The documents may come from teaching and research institutions in France or abroad, or from public or private research centers.
L'archive ouverte pluridisciplinaire HAL, est destinée au dépôt et à la diffusion de documents scientifiques de niveau recherche, publiés ou non, émanant des établissements d'enseignement et de recherche français ou étrangers, des laboratoires publics ou privés. 


\title{
A 1D Continuum Model for Beams with Pantographic Microstructure: Asymptotic Micro-Macro Identification and Numerical Results
}

\author{
Emilio Barchiesi, Francesco dell'Isola, Marco Laudato, Luca Placidi \\ and Pierre Seppecher
}

\begin{abstract}
In the standard asymptotic micro-macro identification theory, starting from a De Saint-Venant cylinder, it is possible to prove that, in the asymptotic limit, only flexible, inextensible, beams can be obtained at the macro-level. In the present paper we address the following problem: is it possible to find a microstructure producing in the limit, after an asymptotic micro-macro identification procedure, a continuum macro-model of a beam which can be both extensible and flexible? We prove that under certain hypotheses, exploiting the peculiar features of a pantographic microstructure, this is possible. Among the most remarkable features of the resulting model we find that the deformation energy is not of second gradient type only because it depends, like in the Euler beam model, upon the Lagrangian curvature, i.e. the projection of the second gradient of the placement function upon the normal vector to the deformed line, but also because it depends upon the projection of the second gradient of the placement on the tangent vector to the deformed line, which is the elongation gradient. Thus, a richer set of boundary conditions can be prescribed
\end{abstract}

\author{
E. Barchiesi · F. dell'Isola (凶) \\ Dipartimento di Ingegneria Strutturale e Geotecnica, Università degli Studi di Roma "La \\ Sapienza", Via Eudossiana 18, 00184 Roma, Italy \\ e-mail: fdellisola@gmail.com \\ E. Barchiesi · F. dell'Isola · M. Laudato $\cdot$ L. Placidi · P. Seppecher \\ International Research Center M\&MoCS, Università degli Studi dell'Aquila, Via Giovanni \\ Gronchi 18 - Zona industriale di Pile, 67100 L'Aquila, Italy \\ L. Placidi \\ Facoltà di Ingegneria, Università Telematica Internazionale UNINETTUNO, Corso Vittorio \\ Emanuele II 39, 00186 Roma, Italy \\ F. dell'Isola \\ Dipartimento di Ingegneria Civile, Edile, Ambientale e Architettura, Università degli Studi \\ dell'Aquila, Via Giovanni Gronchi 18 - Zona industriale di Pile, 67100 L'Aquila, Italy \\ M. Laudato \\ Dipartimento di Ingegneria e Scienze dell'Informazione e Matematica, \\ Università degli Studi dell'Aquila, Via Vetoio 1, 67100 L'Aquila, Coppito, Italy \\ P. Seppecher \\ Institut de Mathématiques de Toulon, Universitè de Toulon et du Var, \\ Avenue de 1'Université, BP 132, Cedex, 83957 La Garde, France
}


for the pantographic beam model. Phase transition and elastic softening are exhibited as well. Using the resulting planar 1D continuum limit homogenized macro-model, by means of FEM analyses, we show some equilibrium shapes exhibiting highly non-standard features. Finally, we conceive that pantographic beams may be used as basic elements in double scale metamaterials to be designed in future.

Keywords Micro-macro identification - Asymptotic expansion - Pantographic beams $\cdot$ Continuum models

\section{Introduction}

Customarily, the theory of nonlinear beams is either postulated by means of a suitable least action principle in the so called "direct way" or is deduced, by means of a more or less rigorous procedure, starting from a three-dimensional elasticity theory. The first example of direct model can be found in the original paper by Euler [1]. Many epigones of Euler used this approach: a comprehensive account for this procedure can be found in e.g. Antman [2]. On the other hand, by following the procedure described by De Saint-Venant, one can try to identify the constitutive equation of an Euler type (1D) model in terms of the geometrical and mechanical properties, at micro-level, of the considered mechanical systems. This is done, in more modern textbooks, by using a more or less standard asymptotic micro-macro identification procedure, which generalizes the one used by De Saint-Venant for bodies with cylindrical shape (see for instance [3]). It can be rigorously proven, under a series of well-precised assumptions, that only flexible and inextensible beams can be obtained [4-9]. In the present paper we address the following problem: is it possible to find a microstructure producing, at the macro level and under loads of the same order of magnitude, a beam which can be both extensible and flexible? Using an asymptotic expansion and rescaling suitably the involved stiffnesses, we prove that a pantographic microstructure does induce, at the macro level, the aforementioned desired mechanical behaviour. In this paper, in an analogous fashion to that of variational asymptotic methods, and following a mathematical approach resembling that used by Piola, we have employed asymptotic expansions of kinematic descriptors directly into the postulated energy functional. Using the so obtained 1D continuum model, we show some equilibrium shapes exhibiting highly non standard features, essentially related to the complete dependence of the homogenized continuum energy density functional on the second gradient of the placement field. While in the standard finite deformation Euler beam theory the energy functional depends only on the material curvature, i.e. the normalized projection of the second gradient of the placement on the normal vector to the current configuration, the energy functional for the nearly-inextensible pantographic beam model depends also on the projection of the second gradient of the placement on the tangent vector to the current configuration. Thus, the full decomposition of the second gradient of the placement is present in the latter model. Generalized continua [10-14], and in particular higher 
gradient theories, see [15] or [16] for a comprehensive review, are able to describe behaviours which cannot be accounted for in classical Cauchy theories [17-24]. In the literature, several examples can be found motivating the importance of generalized continua: electromechanical [25] and biomechanical [26-29] applications, elasticity theory [30-35], capillary fluids analysis [36], granular micromechanics [37-39], robotic systems analysis [40, 41], damage theory [42-47], and wave propagation analysis [48-52]. Furthermore, second gradient continuum models always appear when the considered micro-system is a pantographic structure [53-60]. A comprehensive review on the modeling of pantographic structures can be found in [61, 62]. Several results of numerical investigations can be found in [30, 63-71], while for an outline of recent experimental results we refer to $[72,73]$. The work is organized in the following way. In Sect. 2, we discuss the geometry of the pantographic beam micromechanical model. Once the general expression for the micromodel energy is given, we restrict to the quasi-inextensibility case, where small elongation of oblique fibers is assumed. The micro-model energy is then represented as a function of the macroscopic kinematical descriptors and the further specialization to the (complete) inextensibility of the oblique fibers is considered. In Sect. 3 we perform a heuristic homogenization procedure and we discuss the feature of the 1D continuum model. In particular, we show that such a homogenization procedure gives rise to a full second gradient theory. In Sect. 4 we show results of numerical simulations in order to better highlight some non-standard features of the nearlyinextensible pantographic beam model. Finally, in Sect. 5 we postulate a generalized strain energy density which includes both the quasi-inextensible pantographic beam model and the standard Euler beam theory. Euler-Lagrange equations for this generalized strain energy density are derived together with the corresponding boundary conditions and the specializations to the two models are performed.

\section{Discrete Micro-model}

In this section we discuss the discrete micro-mechanical model which is employed throughout this paper. We begin giving a geometrical description and then we give a mechanical characterization, by choosing a deformation energy. It is a Henckytype spring model with the geometrical arrangement of a pantographic strip. Once the energy of the micro-model is chosen in its general form, we assume a particular asymptotic behaviour for some relevant kinematic quantities, i.e. the elongation of oblique springs, as will be clear in the sequel. We consider the quasi-inextensibility case, i.e. the relative elongation of the oblique springs is small. As a further specialisation, the inextensibility case is considered. Finally, after having defined a micro-macro identification, we express the energy of the micro-system in terms of macroscopic kinematic descriptors to prepare the field to the homogenization procedure which will be discussed in details in the next section. 


\subsection{Geometry}

In the spirit of $[55,59,74]$, in this section we introduce a discrete-spring model (also referred to as the micro-model, since it resembles the features of a specific microstructure). The topology and features of the undeformed and deformed discretespring system are summarized in Figs. 1 and 2, respectively. In the undeformed configuration $N+1$ material particles are arranged upon a straight line at positions $P_{i}$ 's, $i \in[0 ; N]$, with a uniform spacing $\varepsilon$. The basic $i$-th unit cell centered in $P_{i}$ is formed by four springs joined together by a hinge placed at $P_{i}$. Between two oblique springs, belonging to the same cell and lying on the same diagonal, a rotational spring opposing to their relative rotation is placed. Rotational springs are colored in Fig. 1 in blue and red.

We denote with $p_{i}$ the position in the deformed configuration corresponding to position $P_{i}$ in the reference one. In order to completely describe the kinematics of the micro-model we have to introduce other descriptors. At this end, the length of the oblique deformed springs, indicated with $l_{i}^{\alpha \beta}$, is introduced, the indices $\alpha$ and $\beta$ belonging respectively to the sets $\{1,2\}$ and $\{D, S\}$ and referring to the first and

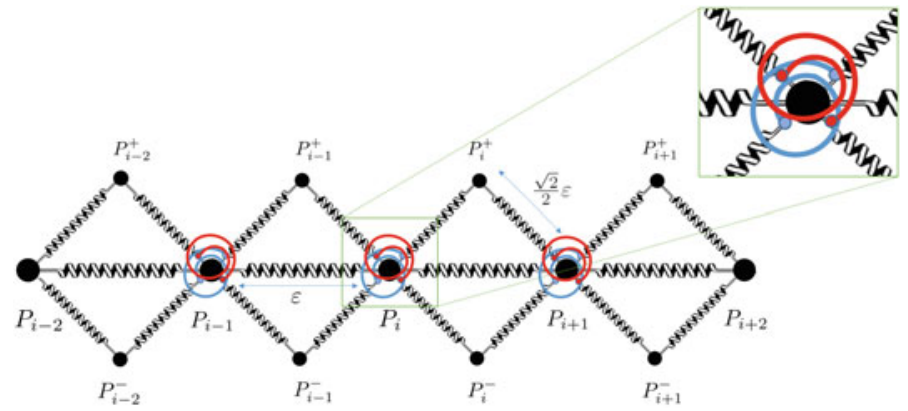

Fig. 1 Undeformed spring system resembling the micro-structure

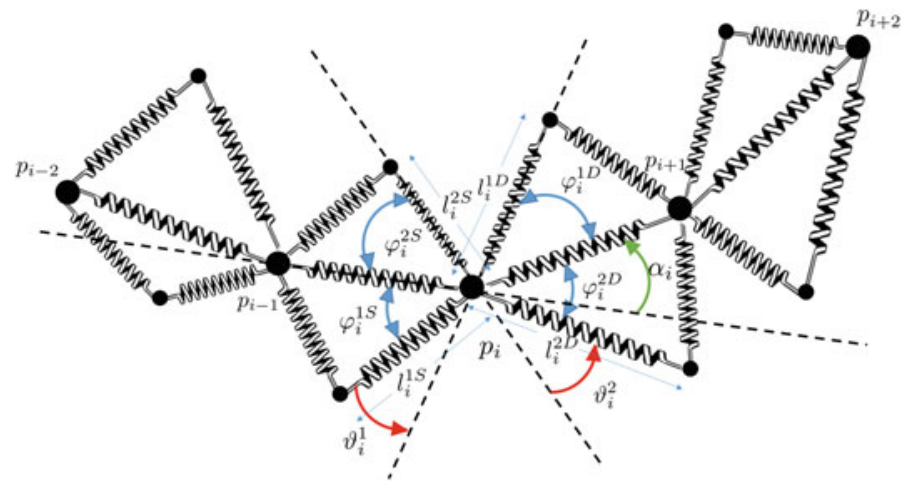

Fig. 2 Deformed spring system resembling the micro-structure 
second diagonal and left and right, respectively. Referring to Fig. 2, we consider the $i$-th node, notwithstanding that the same quantities can be defined for each node. We define $\alpha_{i}$ as the angle between the vectors $p_{i}-p_{i-1}$ and $p_{i}-p_{i+1}$, respectively. We define as $\vartheta_{i}^{\alpha}$ the angle measuring the deviation of two opposite oblique springs from being collinear. In order to illustrate the definition of $\varphi_{i}^{\alpha \beta}$, we consider the case $\alpha=1$ and $\beta=D$. The quantity $\varphi_{i}^{1 D}$ is the angle between the vector $p_{i+1}-p_{i}$ and the upper oblique spring hinged at $p_{i}$. By means of elementary geometric considerations, we have that

$$
\begin{aligned}
& \vartheta_{i}^{1}=\alpha_{i}+\varphi_{i}^{1 D}-\varphi_{i}^{1 S} \\
& \vartheta_{i}^{2}=\alpha_{i}+\varphi_{i}^{2 S}-\varphi_{i}^{2 D}, i \in[0 ; N] .
\end{aligned}
$$

In the undeformed configuration, see Fig. 1, we have:

$$
\begin{aligned}
& l_{i}^{\alpha \beta}=\frac{\sqrt{2}}{2} \varepsilon, \alpha=1,2 \quad \beta=D, S \quad i \in[0 ; N] \\
& \vartheta_{i}^{1}=\vartheta_{i}^{2}=0 \\
& \left\|p_{i}-p_{i-1}\right\|=\varepsilon, i \in[0 ; N] .
\end{aligned}
$$

Considering that $\varphi_{i}^{\alpha D}, \varphi_{i}^{\alpha S} \in[0, \pi]$, by means of the law of cosines, we get:

$$
\begin{aligned}
& \varphi_{i}^{1 D}=\cos ^{-1}\left(\frac{\left\|p_{i+1}-p_{i}\right\|^{2}+\left(l_{i}^{1 D}\right)^{2}-\left(l_{i+1}^{2 S}\right)^{2}}{2 l_{i}^{1 D}\left\|p_{i+1}-p_{i}\right\|}\right) \\
& \varphi_{i}^{2 D}=\cos ^{-1}\left(\frac{\left\|p_{i+1}-p_{i}\right\|^{2}+\left(l_{i}^{2 D}\right)^{2}-\left(l_{i+1}^{1 S}\right)^{2}}{2 l_{i}^{2 D}\left\|p_{i+1}-p_{i}\right\|}\right) \\
& \varphi_{i}^{1 S}=\cos ^{-1}\left(\frac{\left\|p_{i}-p_{i-1}\right\|^{2}+\left(l_{i}^{1 S}\right)^{2}-\left(l_{i-1}^{2 D}\right)^{2}}{2 l_{i}^{1 S}\left\|p_{i}-p_{i-1}\right\|}\right) \\
& \varphi_{i}^{2 S}=\cos ^{-1}\left(\frac{\left\|p_{i}-p_{i-1}\right\|^{2}+\left(l_{i}^{2 S}\right)^{2}-\left(l_{i-1}^{1 D}\right)^{2}}{2 l_{i}^{2 S}\left\|p_{i}-p_{i-1}\right\|}\right) .
\end{aligned}
$$




\subsection{Mechanical Model}

The micro model energy, written as a combination of the elastic energy contributions of the springs, is defined as:

$$
\begin{aligned}
& \mathcal{M}= \\
& \sum_{i} \sum_{\alpha, \beta} \frac{k_{\alpha \beta, i}^{e}}{2}\left(l_{i}^{\alpha \beta}-\frac{\sqrt{2}}{2} \varepsilon\right)^{2}+\sum_{i} \sum_{\alpha} \frac{k_{\alpha, i}^{f}}{2}\left(\vartheta_{i}^{\alpha}\right)^{2}+ \\
& +\sum_{i} \frac{k_{i}^{m}}{2}\left(\left\|p_{i+1}-p_{i}\right\|-\varepsilon\right)^{2}=
\end{aligned}
$$

Reminding that $\vartheta_{i}^{\alpha}=\alpha_{i}+(-1)^{\alpha}\left(\varphi_{i}^{\alpha S}-\varphi_{i}^{\alpha D}\right)$, then (4) recasts as:

$$
\begin{aligned}
& \mathcal{M}= \\
& \sum_{i} \sum_{\alpha, \beta} \frac{k_{\alpha \beta, i}^{e}}{2}\left(l_{i}^{\alpha \beta}-\frac{\sqrt{2}}{2} \varepsilon\right)^{2}+\sum_{i} \sum_{\alpha} \frac{k_{\alpha, i}^{f}}{2}\left[\alpha_{i}+(-1)^{\alpha}\left(\varphi_{i}^{\alpha S}-\varphi_{i}^{\alpha D}\right)\right]^{2}+ \\
& +\sum_{i} \frac{k_{i}^{m}}{2}\left(\left\|p_{i+1}-p_{i}\right\|-\varepsilon\right)^{2} .
\end{aligned}
$$

In the next subsections we will specialize this form of the energy by means of assumptions on the properties of the micro-system. In particular, we will discuss in detail the representation of the micro-energy for the quasi-inextensibility assumption that will be made clear next and, subsequently, for the (complete) inextensibility cases.

\subsection{Asymptotic Expansion and Quasi-inextensibility Assumption}

We postulate that the following asymptotic expansion holds for $l_{i}^{\alpha \beta}$

$$
l_{i}^{\alpha \beta}=\varepsilon \tilde{l}_{i 1}^{\alpha \beta}+\varepsilon^{2} \tilde{l}_{i 2}^{\alpha \beta}+o\left(\varepsilon^{2}\right),
$$

where the constant (with respect to $\varepsilon$ ) term is not present. We now turn to what we refer to as the quasi-inextensibility case. It consists in fixing the value of the firstorder term in (6) as $\tilde{l}_{i 1}^{\alpha \beta}=\frac{\sqrt{2}}{2}$. Moreover, to lighten the notation, we drop the subscript "2" of $\tilde{l}_{i 2}^{\alpha \beta}$ i.e. $\tilde{l}_{i}^{\alpha \beta}:=\tilde{l}_{i 2}^{\alpha \beta}$. Hence, (6) reads as: 


$$
l_{i}^{\alpha \beta}=\frac{\sqrt{2}}{2} \varepsilon+\varepsilon^{2} \tilde{l}_{i}^{\alpha \beta}+o\left(\varepsilon^{2}\right) .
$$

\subsection{Piola's Ansatz}

The reference shape of the macro-model is a one-dimensional straight segment $S$ and we introduce on it an abscissa $s \in[0, B]$ - where $B=N \varepsilon$ is the length of $S$ which labels each position in $S$. Proceeding as in the pioneering works of Gabrio Piola, an Italian mathematician and physicist who lived in the 1800s (see [75] for a historical review), we introduce the so-called kinematical maps, i.e. some fields in the macro-model that uniquely determine $p_{i}$ and $\tilde{l}_{i}^{\alpha \beta}$ :

$$
\begin{gathered}
\chi:[0, B] \rightarrow \mathcal{E} \\
\tilde{l}^{\alpha \beta}:[0, B] \rightarrow \mathbb{R}^{+},
\end{gathered}
$$

with $\mathcal{E}$ the Euclidean space on $\mathbb{V} \equiv \mathbb{R}^{2}$. We choose $\chi$ to be the placement function of the $1 \mathrm{D}$ continuum and, hence, it has to be injective. The current shape can be regarded as the image of the (sufficiently smooth) curve $\chi:[0, B] \rightarrow \mathcal{E}$ and, unlike the reference shape, it is not parameterized by its arc-length and it is not a straight line in general. In order for these fields to uniquely determine the kinematical descriptors of the micro-model (i.e. $p_{i}$ and $\tilde{l}_{i}^{\alpha \beta}$ ), we use the Piola's ansatz and impose

$$
\begin{aligned}
& \chi\left(s_{i}\right)=p_{i} \\
& \tilde{l}^{\alpha \beta}\left(s_{i}\right)=\tilde{l}_{i}^{\alpha \beta}, \forall i \in[0 ; N] .
\end{aligned}
$$

\subsection{Micro-model Energy as a Function of Macro-model Descriptors}

In this subsection we obtain the micro-model energy for the quasi-inextensibility case in terms of the macroscopic kinematical maps. Assuming that $\chi$ is at least twice continuously differentiable with respect to the space variable in $s_{i}$ 's, we have

$$
\begin{aligned}
& \chi\left(s_{i+1}\right)=\chi\left(s_{i}\right)+\varepsilon \chi^{\prime}\left(s_{i}\right)+\frac{\varepsilon^{2}}{2} \chi^{\prime \prime}\left(s_{i}\right)+o\left(\varepsilon^{2}\right) \\
& \chi\left(s_{i-1}\right)=\chi\left(s_{i}\right)-\varepsilon \chi^{\prime}\left(s_{i}\right)+\frac{\varepsilon^{2}}{2} \chi^{\prime \prime}\left(s_{i}\right)+o\left(\varepsilon^{2}\right) .
\end{aligned}
$$


Plugging (9) in (7) and (10), we get the following expressions:

$$
\begin{aligned}
& l_{i}^{\alpha \beta}=\frac{\sqrt{2}}{2} \varepsilon+\varepsilon^{2} \tilde{l}^{\alpha \beta}\left(s_{i}\right)+o\left(\varepsilon^{2}\right) \\
& p_{i+1}-p_{i}=\varepsilon \chi^{\prime}\left(s_{i}\right)+\frac{\varepsilon^{2}}{2} \chi^{\prime \prime}\left(s_{i}\right)+o\left(\varepsilon^{2}\right) \\
& p_{i-1}-p_{i}=-\varepsilon \chi^{\prime}\left(s_{i}\right)+\frac{\varepsilon^{2}}{2} \chi^{\prime \prime}\left(s_{i}\right)+o\left(\varepsilon^{2}\right) .
\end{aligned}
$$

Substituting (11) into (3) and expanding $\varphi_{i}^{\alpha S}-\varphi_{i}^{\alpha D}$ up to first-order with respect to $\varepsilon$, we get

$$
\begin{aligned}
& \varphi_{i}^{\alpha S}-\varphi_{i}^{\alpha D}= \\
& \frac{\frac{\sqrt{2}}{4}\left[\left\|\chi^{\prime}\left(s_{i}\right)\right\|^{2}\right]^{\prime}+\left[\tilde{l}^{(3-\alpha) D}\left(s_{i-1}\right)-\tilde{l}^{(3-\alpha) S}\left(s_{i+1}\right)\right]}{\left\|\chi^{\prime}\left(s_{i}\right)\right\| \sqrt{1-\frac{\left\|\chi^{\prime}\left(s_{i}\right)\right\|^{2}}{2}}} \varepsilon+ \\
& +\frac{\left[\left\|\chi^{\prime}\left(s_{i}\right)\right\|^{2}-1\right]\left[\tilde{l}^{\alpha S}\left(s_{i}\right)-\tilde{l}^{\alpha D}\left(s_{i}\right)\right]}{\left\|\chi^{\prime}\left(s_{i}\right)\right\| \sqrt{1-\frac{\left\|\chi^{\prime}\left(s_{i}\right)\right\|^{2}}{2}}} \varepsilon+o(\varepsilon) .
\end{aligned}
$$

Finally, substituting (12) in (5) yields the micro-model energy $\mathcal{M}$ as a function of the kinematical descriptors $\chi$ and $\tilde{l}^{\alpha \beta}$ of the macro-model

$$
\begin{aligned}
& \mathcal{M}= \\
& \sum_{i} \sum_{\alpha, \beta} \frac{k_{\alpha \beta, i}^{e} \varepsilon^{4}}{2}\left(\tilde{l}_{i}^{\alpha \beta}\right)^{2}+\sum_{i} \frac{k_{i}^{m} \varepsilon^{2}}{2}\left(\left\|\chi_{i}^{\prime}\right\|-1\right)^{2}+ \\
& +\sum_{i} \sum_{\alpha} \frac{k_{\alpha, i}^{f} \varepsilon^{2}}{2}\left\{\vartheta^{\prime}\left(s_{i}\right)+(-1)^{\alpha} \frac{\frac{\sqrt{2}}{4}\left[\left\|\chi^{\prime}\left(s_{i}\right)\right\|^{2}\right]^{\prime}+\left[\tilde{l}_{i}^{(3-\alpha) D}\left(s_{i-1}\right)-\tilde{l}_{i}^{(3-\alpha) S}\left(s_{i+1}\right)\right]}{\left\|\chi^{\prime}\left(s_{i}\right)\right\| \sqrt{1-\frac{\left\|\chi^{\prime}\left(s_{i}\right)\right\|^{2}}{2}}+}\right. \\
& +(-1)^{\alpha} \frac{\left[\left\|\chi^{\prime}\left(s_{i}\right)\right\|^{2}-1\right]\left[\tilde{l}_{i}^{\alpha S}\left(s_{i}\right)-\tilde{l}_{i}^{\alpha D}\left(s_{i}\right)\right]}{\left.\left\|\chi^{\prime}\left(s_{i}\right)\right\| \sqrt{1-\frac{\left\|\chi^{\prime}\left(s_{i}\right)\right\|^{2}}{2}}\right\}^{2},}
\end{aligned}
$$

where $\alpha_{i}=\varepsilon \vartheta^{\prime}\left(s_{i}\right)$ has been used and

$$
\vartheta^{\prime}=\frac{\chi_{\perp}^{\prime} \cdot \chi^{\prime \prime}}{\left\|\chi^{\prime}\right\|^{2}}
$$


with $\chi_{\perp}^{\prime}$ the $90^{\circ}$ anti-clockwise rotation of $\chi^{\prime}$, is the material curvature i.e. rate of change with respect to the reference abscissa of the orientation of the tangent $\chi^{\prime}(s)=\rho(s)\left[\cos \vartheta(s) \mathbf{e}_{1}+\sin \vartheta(s) \mathbf{e}_{2}\right]$ to the deformed centerline. We remark that the micro-model energy, when written in terms of macroscopic fields, contains already a contribution from the second gradient of $\chi(s)$. Finally, it is worth to be noticed that, for a fixed $\varepsilon$, Eq. (13) provides an upper bound for $\left\|\chi^{\prime}\right\|$, i.e. $\left\|\chi^{\prime}\right\|<\sqrt{2}$ , even if no kinematic restrictions directly affect $\left\|\chi^{\prime}\right\|$.

\subsection{The Inextensibility Case}

We consider now the case of inextensible oblique springs. This translates in considering $\tilde{l}_{i}^{\alpha \beta}=0$ and it is referred as the inextensibility case. Moreover, for the sake of simplicity we consider the elastic constants of the rotational springs to satisfy $k_{1, i}^{f}=k_{2, i}^{f}:=k_{i}^{f}, \forall i \in[1 ; N]$. We remark that $\tilde{l}_{i}^{\alpha \beta}=0$ implies, through a purely geometric argument, that $\varphi_{i+1}^{S 1}=\varphi_{i+1}^{S 2}=\varphi_{i}^{D 1}=\varphi_{i}^{D 2}:=\varphi_{i}$. Once the kinematic restrictions implied by the inextensibility assumption have been presented, we are ready to define the micro-model energy (5) as

$$
\begin{aligned}
& \mathcal{I}= \\
& \sum_{i} k_{i}^{f} \sum_{\alpha} \frac{\left[\alpha_{i}+(-1)^{\alpha}\left(\varphi_{i}-\varphi_{i-1}\right)\right]^{2}}{2}+\sum_{i} \frac{k_{i}^{m}}{2}\left(\left\|p_{i+1}-p_{i}\right\|-\varepsilon\right)^{2} .
\end{aligned}
$$

Proceeding in analogy with the previous construction, we introduce the kinematical map

$$
\varphi:[0, B] \rightarrow\left[0, \frac{\pi}{2}\right]
$$

and, then, we perform the Piola's ansatz by imposing

$$
\varphi\left(s_{i}\right)=\varphi_{i}, \forall i \in[0 ; N] .
$$

Assuming both $\chi$ and $\varphi$ to be at least one time continuously differentiable with respect to the space variable in $s_{i}$ and taking into account the Piola's ansatz (15), we have

$$
\begin{aligned}
& p_{i+1}-p_{i}=\varepsilon \chi^{\prime}\left(s_{i}\right)+o(\varepsilon) \\
& \varphi_{i-1}-\varphi_{i}=-\varepsilon \varphi^{\prime}\left(s_{i}\right)+o(\varepsilon) .
\end{aligned}
$$


Substituting (16) into (14) yields the micro-model energy for the inextensibility case in terms of the kinematical quantities of the macro-model

$$
\begin{aligned}
& \mathcal{I}= \\
& \sum_{i} k_{i}^{f} \varepsilon^{2}\left[\vartheta^{\prime 2}\left(s_{i}\right)+\varphi_{i}^{\prime 2}\left(s_{i}\right)\right]+\sum_{i} \frac{k_{i}^{m} \varepsilon^{2}}{2}\left(\left\|\chi_{i}^{\prime}\right\|-1\right)^{2} .
\end{aligned}
$$

We now impose the so-called internal connection constraint:

$$
\sqrt{2} \varepsilon \cos \varphi\left(s_{i}\right)=\left\|\chi\left(s_{i+1}\right)-\chi\left(s_{i}\right)\right\|,
$$

which, up to $\varepsilon$-terms of order higher than one, reads:

$$
\sqrt{2} \cos \varphi=\left\|\chi^{\prime}\right\| \text {. }
$$

This constraint ensures that, in the deformed configuration, the upper-left spring of the $i$-th cell is hinge-joint with the upper-right spring of the $(i-1)$-th cell, and the lower-left spring of the $i$-th cell is hinge-joint with lower-right spring of the $(i-1)$-th cell. Due to this constraint, the maps $\varphi$ and $\chi$ are not independent and it is possible to rewrite the expression of the micro-model energy in terms of the placement field $\chi(s)$ only. Indeed, deriving (19) with respect to the space variable yields

$$
-\sqrt{2} \varphi^{\prime}\left(s_{i}\right) \sin \varphi\left(s_{i}\right)=\left\|\chi^{\prime}\left(s_{i}\right)\right\|^{\prime},
$$

which, in turn, implies

$$
\varphi^{\prime}\left(s_{i}\right)=-\frac{\left\|\chi^{\prime}\left(s_{i}\right)\right\|^{\prime}}{\sqrt{2} \sin \varphi\left(s_{i}\right)} .
$$

Reminding $\varphi \in[0, \pi]$ and taking into account (19), we get:

$$
\begin{aligned}
& \varphi_{i}^{\prime}=-\frac{\left\|\chi_{i}^{\prime}\right\|^{\prime}}{\sqrt{2} \sqrt{1-\cos ^{2} \gamma_{i}}}= \\
& =-\frac{\left\|\chi_{i}^{\prime}\right\|^{\prime}}{\sqrt{2-\left\|\chi_{i}^{\prime}\right\|^{2}}} .
\end{aligned}
$$

Hence, in the inextensibility case, the micro-model energy (17) can be recast, as a function of the macro-model descriptor $\chi$ only, as 


$$
\begin{aligned}
& \mathcal{I}= \\
& \sum_{i} k_{i}^{f} \varepsilon^{2}\left[\left[\vartheta^{\prime}\left(s_{i}\right)\right]^{2}+\left(\frac{\left\|\chi^{\prime}\left(s_{i}\right)\right\|^{\prime}}{\sqrt{2-\left\|\chi^{\prime}\left(s_{i}\right)\right\|^{2}}}\right)^{2}\right]+\sum_{i} \frac{k_{i}^{m} \varepsilon^{2}}{2}\left(\left\|\chi^{\prime}\left(s_{i}\right)\right\|-1\right)^{2}
\end{aligned}
$$

Clearly, since the inextensibility case is just a special case of the quasi-inextensibility case, it is possible to show that this expression can be also obtained in a more direct way from (13) by setting $\tilde{l}^{\alpha S}\left(s_{i}\right)=0$ and $k_{1, i}^{f}=k_{2, i}^{f}:=k_{i}^{f}$.

\section{Continuum-Limit Macro-model}

In this section, by performing the final steps of the heuristic homogenization procedure presented throughout this paper, we derive a 1D continuum model, also referred to as the macro-model, associated to the aforementioned micro-structure. Besides, we analyse the quasi-inextensibility and inextensibility cases and we obtain the corresponding macro-model energies in terms of the displacement field $\chi$.

\subsection{Rescaling of Stiffnesses and Heuristic Homogenization}

The preliminary step to perform the homogenization procedure consists into the definition of the quantities $\mathbb{K}_{\alpha \beta, i}^{e}, \mathbb{K}_{\alpha, i}^{f}$ and $\mathbb{K}_{i}^{m}$. These quantities are scale invariant, meaning that they do not depend on $\varepsilon$. Their role is to keep track of the asymptotic behaviour of the stiffnesses $k_{\alpha, \beta, i}^{e}, k_{\alpha, i}^{f}$, and $k_{i}^{m}$ of the micro-model springs. More explicitly, we assume:

$$
k_{\alpha \beta, i}^{e}(\varepsilon)=\frac{\mathbb{K}_{\alpha \beta, i}^{e} ;}{\varepsilon^{3}} ; \quad k_{\alpha, i}^{f}(\varepsilon)=\frac{\mathbb{K}_{\alpha, i}^{f}}{\varepsilon} ; \quad k_{i}^{m}(\varepsilon)=\frac{\mathbb{K}_{i}^{m}}{\varepsilon} .
$$

We remark that in this rescaling, as $\varepsilon$ approaches zero, the ratio between the stiffness $k_{\alpha \beta, i}^{e}$ of the oblique springs and the stiffness $k_{\alpha, i}^{f}$ will approach infinity with a rate of divergence in $\varepsilon$ equal to two, i.e. $\frac{k_{\alpha \beta, i}^{e}}{k_{\alpha, i}^{e}} \sim \varepsilon^{2}$. Now, we are ready to perform the homogenization procedure. Firstly, we consider the more general quasi-inextensibility case. For simplicity, let us set

$$
\mathbb{K}_{1 D, i}^{e}=\mathbb{K}_{1 S, i}^{e}=\mathbb{K}_{2 D, i}^{e}=\mathbb{K}_{2 S, i}^{e}:=\mathbb{K}_{i}^{e} ; \quad \mathbb{K}_{1, i}^{f}=\mathbb{K}_{2, i}^{f}:=\mathbb{K}_{i}^{f} .
$$

Let us introduce the kinematical maps 


$$
\mathbb{K}^{e}:[0, B] \rightarrow \mathbb{R}^{+} ; \quad \mathbb{K}^{f}:[0, B] \rightarrow \mathbb{R}^{+} ; \quad \mathbb{K}^{m}:[0, B] \rightarrow \mathbb{R}^{+}
$$

such that they satisfy the following Piola's ansatz:

$$
\mathbb{K}^{e}\left(s_{i}\right)=\mathbb{K}_{i}^{e} ; \quad \mathbb{K}^{f}\left(s_{i}\right)=\mathbb{K}_{i}^{f} ; \quad \mathbb{K}^{m}\left(s_{i}\right)=\mathbb{K}_{i}^{m} .
$$

Substituting (22) in (13), taking into account (23) and (24), and letting $\varepsilon \rightarrow 0$ yield

$$
\begin{aligned}
& \mathcal{E}= \\
& \int_{S} \frac{\mathbb{K}^{e}}{2}\left(\tilde{l}^{1 S}\right)^{2} \mathrm{ds}+\int_{S} \frac{\mathbb{K}^{e}}{2}\left(\tilde{l}^{1 D}\right)^{2} \mathrm{ds}+\int_{S} \frac{\mathbb{K}^{e}}{2}\left(\tilde{l}^{2 S}\right)^{2} \mathrm{ds}+\int_{S} \frac{\mathbb{K}^{e}}{2}\left(\tilde{l}^{2 D}\right)^{2} \mathrm{ds}+ \\
& +\int_{S} \frac{\mathbb{K}^{f}}{2}\left\{\vartheta^{\prime}+\frac{-\sqrt{2}\left(\left\|\chi^{\prime}\right\|^{2}\right)^{\prime}-4\left[\left(\tilde{l}^{2 D}-\tilde{l}^{2 S}\right)-\left(\left\|\chi^{\prime}\right\|^{2}-1\right)\left(\tilde{l}^{1 D}-\tilde{l}^{1 S}\right)\right]}{\left\|\chi^{\prime}\right\| \sqrt{2-\left\|\chi^{\prime}\right\|^{2}}}\right\}^{2} \mathrm{ds}+ \\
& +\int_{S} \frac{\mathbb{K}^{f}}{2}\left\{\vartheta^{\prime}+\frac{\sqrt{2}\left(\left\|\chi^{\prime}\right\|^{2}\right)^{\prime}+4\left[\left(\tilde{l}^{1 D}-\tilde{l}^{1 S}\right)+\left(\left\|\chi^{\prime}\right\|^{2}-1\right)\left(\tilde{l}^{S S}-\tilde{l}^{2 D}\right)\right]}{\left\|\chi^{\prime}\right\| \sqrt{2-\left\|\chi^{\prime}\right\|^{2}}}\right\}^{2} \mathrm{ds}+ \\
& +\int_{S} \frac{\mathbb{K}^{m}}{2}\left(\left\|\chi^{\prime}\right\|-1\right)^{2} \mathrm{ds} .
\end{aligned}
$$

which is the continuum-limit macro-model energy for a 1D pantographic beam under the hypothesis of quasi-inextensible oblique micro-springs. It is worth to remark that, when $\mathbb{K}^{m}=0, \tilde{l}^{\alpha \beta}=0$ and $\chi(s)=C s \mathbf{e}_{1}$, with $C \in \mathbb{R}$, the beam undergoes a floppy mode i.e. (25) vanishes. Thus, under the above conditions, the configuration $\chi(s)=C s \mathbf{e}_{1}$ is isoenergetic to the undeformed configuration for any $C$. For a fixed $\varepsilon$, considering $k_{i}^{m}=0$ and $\tilde{l}_{i}^{\alpha \beta}=0$ in the micro-model energy (13), we have that $\chi\left(s_{i}\right)=C s_{i} \mathbf{e}_{1}$ is a floppy mode for the micro-model as well. This means that the homogenization procedure that we have carried out has preserved a key feature of the micro-model. Up to now, the expression of the continuum limit homogenized energy depends both on the kinematical maps $\chi$ and $\tilde{l}$. In the next section we show that, at equilibrium, it is possible to write the macro-energy in terms of the placement field only.

\subsection{Macro-model Energy as a Function of the Placement field}

We now equate to zero the first variations of (25) with respect to $\tilde{l}^{\alpha \beta}$, s, i.e. we look for stationary points, with respect to $\tilde{l}^{\alpha \beta}$, of (25). This is a necessary first order condition 
for optimality. In the continuum limit homogenized energy no spatial derivatives of $\tilde{l}^{\alpha \beta}$ appear. Such energy depends only by linear and quadratic contributions in $\tilde{l}^{\alpha \beta}$. Hence, this process yields four algebraic linear equations in $\tilde{l}^{\alpha \beta}$. Solving these equations gives $\tilde{l}^{\alpha \beta}$ at equilibrium

$$
\begin{aligned}
& \tilde{l}^{1 D}=\frac{\sqrt{2}}{2} \mathbb{K}^{f}\left(\chi^{\prime \prime} \cdot C+\vartheta^{\prime} D\right) \\
& \tilde{l}^{2 D}=\frac{\sqrt{2}}{2} \mathbb{K}^{f}\left(\chi^{\prime \prime} \cdot C-\vartheta^{\prime} D\right) \\
& \tilde{l}^{1 S}=\frac{\sqrt{2}}{2} \mathbb{K}^{f}\left(-\chi^{\prime \prime} \cdot C-\vartheta^{\prime} D\right) \\
& \tilde{l}^{2 S}=\frac{\sqrt{2}}{2} \mathbb{K}^{f}\left(-\chi^{\prime \prime} \cdot C+\vartheta^{\prime} D\right)
\end{aligned}
$$

with

$$
\begin{aligned}
& C=\frac{\chi^{\prime}}{2 \mathbb{K} f\left\|\chi^{\prime}\right\|^{2}-\frac{1}{2}\left(\mathbb{\mathbb { K } ^ { e }}\left\|\chi^{\prime}\right\|^{2}+8 \mathbb{K}^{f}\right)} \\
& D=\frac{\left\|\chi^{\prime}\right\| \sqrt{4 \tilde{L}^{2}-\left\|\chi^{\prime}\right\|^{2}}}{\mathbb{K}^{e} \tilde{L}^{2}\left(\left\|\chi^{\prime}\right\|^{2}-2\right)-2 \mathbb{K}^{f}\left\|\chi^{\prime}\right\|^{2}} .
\end{aligned}
$$

From (26) we can get, in some particular cases, interesting information about the properties of the pantographic beam. Firstly, let us notice that $\tilde{l}^{1 D}=-\tilde{l}^{1 S}$ and $\tilde{l}^{2 D}=$ $-\tilde{l}^{2 S}$. Moreover, we also notice that when $\chi^{\prime}=\rho \mathbf{e}_{1}$, with $\rho$ independent of the abscissa $s$, then, as $\chi^{\prime \prime}$ vanishes, $\tilde{l}^{\alpha \beta}=0$ i.e. the fibers undergo no elongation. Instead, when $\chi^{\prime}(s)=\rho(s) \mathbf{e}_{1}$, with $\rho$ depending on $s$, then $\tilde{l}^{1 D}=\tilde{l}^{2 D}=-\tilde{l}^{1 S}=-\tilde{l}^{2 S}$. This remarkable and counter-intuitive feature can be used as a possible benchmark test to validate, as $\varepsilon$ approaches zero, a numerical scheme based on the discrete micro-model. Let us consider the case of non-zero bending curvature, i.e. $\vartheta^{\prime} \neq 0$, when $\chi^{\prime \prime} \cdot C<<\vartheta^{\prime} D$, which implies that $\tilde{l}^{1 D}=-\tilde{l}^{2 D}=-\tilde{l}^{1 S}=\tilde{l}^{2 S}$. If $\vartheta^{\prime}>0$ then $\tilde{l}^{1 D}, \tilde{l}^{2 S}>0$ and $\tilde{l}^{2 D}, \tilde{l}^{1 S}<0$ while, if $\vartheta^{\prime}<0$ then $\tilde{l}^{1 D}, \tilde{l}^{2 S}<0$ and $\tilde{l}^{2 D}, \tilde{l}^{1 S}>0$. We are now ready to express the macro-model energy $\overline{\mathcal{E}}(\chi)$ as a function of the placement $\chi$ only, by substituting (26) in (25): 


$$
\begin{aligned}
& \overline{\mathcal{E}}(\chi(\cdot))=\min _{\tilde{I}^{\alpha \beta}(\cdot)} \mathcal{E}= \\
& \int_{S} \mathbb{K}^{e} \mathbb{K}^{f}\left\{\frac{\left(\rho^{2}-2\right)}{\rho^{2}\left(\mathbb{K}^{e}-4 \mathbb{K}^{f}\right)-2 \mathbb{K}^{e}} \vartheta^{\prime 2}+\frac{\rho^{2}}{\left(2-\rho^{2}\right)\left[\rho^{2}\left(\mathbb{K}^{e}-4 \mathbb{K}^{f}\right)+8 \mathbb{K}^{f}\right]} \rho^{\prime 2}\right\} \mathrm{ds}+ \\
& +\int_{S} \frac{\mathbb{K}^{m}}{2}(\rho-1)^{2} \mathrm{ds}= \\
& =\int_{S} \frac{\mathbb{\mathbb { K } ^ { e }} \mathbb{K}^{f}\left(\left\|\chi^{\prime}\right\|^{2}-2\right)}{\left\|\chi^{\prime}\right\|^{4}\left[\left\|\chi^{\prime}\right\|^{2}\left(\mathbb{K}^{e}-4 \mathbb{K}^{f}\right)-2 \mathbb{K} \mathbb{K}^{e}\right]}\left(\chi_{\perp}^{\prime} \cdot \chi^{\prime \prime}\right)^{2} \mathrm{ds}+ \\
& +\int_{S} \frac{\mathbb{K}^{e} \mathbb{K}^{f}}{\left(2-\left\|\chi^{\prime}\right\|^{2}\right)\left[\left\|\chi^{\prime}\right\|^{2}\left(\mathbb{K}^{e}-4 \mathbb{K}^{f}\right)+8 \mathbb{K}^{f}\right]}\left(\chi^{\prime} \cdot \chi^{\prime \prime}\right)^{2} \mathrm{ds}+ \\
& +\int_{S} \frac{\mathbb{K}^{m}}{2}\left(\left\|\chi^{\prime}\right\|-1\right)^{2} \mathrm{ds} .
\end{aligned}
$$

We observe that, for $0<\rho<\sqrt{2}$ and for any choice of the positive macro-stiffnesses $\mathbb{K}^{e}, \mathbb{K}^{f}$ and $\mathbb{K}^{m},(27)$ is positive definite. Moreover, not only we can classify this homogenized model as a second gradient theory, but we notice that the full second gradient $\chi^{\prime \prime}$ of $\chi$ contributes to the strain energy. Indeed, beyond the usual term $\left(\chi_{\perp}^{\prime} \cdot \chi^{\prime \prime}\right)$ related to the Lagrangian curvature, also the term $\left(\chi^{\prime} \cdot \chi^{\prime \prime}\right)$, deriving from the presence of the oblique springs, appears. There is a remarkable feature in this model which deserves to be discussed. From (27), it is clear that in the limit $\left\|\chi^{\prime}\right\| \rightarrow$ $\sqrt{2}$ the model exhibits a so-called phase transition: it locally degenerates into the model of an uniformly extensible cable, notwithstanding that $\sqrt{2}$ is an upper bound for $\rho$. Indeed,

$$
\begin{aligned}
& \frac{\left(\rho^{2}-2\right)}{\rho^{2}\left(\mathbb{K}^{e}-4 \mathbb{K}^{f}\right)-2 \mathbb{K}^{e}} \rightarrow 0 \\
& \frac{\rho^{2}}{\left(2-\rho^{2}\right)\left[\rho^{2}\left(\mathbb{K}^{e}-4 \mathbb{K}^{f}\right)+8 \mathbb{K}^{f}\right]} \rightarrow+\infty,
\end{aligned}
$$

so that no deformation energy is stored for finite bending curvature and, in order for the energy to be bounded for bounded deformations, $\rho^{\prime}$ must approach zero, meaning that the elongation must be locally uniform. Further developments of this model could consist in contemplating a phase transition to a model that, for finite bending curvature, entails a non-zero stored deformation energy.

\subsubsection{Non-dimensionalization}

In order to handle more easily the model in the numerical implementation and in the interpretation of the corresponding results, we turn to the use of non-dimensional 
quantities. Therefore, we introduce the following non-dimensional fields:

$$
s=B \bar{s} ; \quad \chi=B \bar{\chi} ; \quad \mathbb{K}^{e}=K \overline{\mathbb{K}}^{e} ; \quad \mathbb{K}^{f}=K \overline{\mathbb{K}}^{f} ; \quad \mathbb{K}^{m}=K^{m} \overline{\mathbb{K}}^{m} .
$$

In terms of these new quantities, we can recast (27) as

$$
\begin{aligned}
& \frac{K}{B} \int_{0}^{1} \frac{\overline{\mathbb{K}}^{e} \overline{\mathbb{K}}^{f}\left(\left\|\bar{\chi}^{\prime}\right\|^{2}-2\right)}{\left\|\bar{\chi}^{\prime}\right\|^{4}\left[\left\|\bar{\chi}^{\prime}\right\|^{2}\left(\overline{\mathbb{K}}^{e}-4 \overline{\mathbb{K}}^{f}\right)-2 \overline{\mathbb{K}}^{e}\right]}\left(\bar{\chi}_{\perp}^{\prime} \cdot \bar{\chi}^{\bar{\prime}}\right)^{2} \mathrm{~d} \overline{\mathrm{s}}+ \\
& +\frac{K}{B} \int_{0}^{1} \frac{\overline{\mathbb{K}}^{e} \overline{\mathbb{K}}^{f}\left(\bar{\chi}^{\prime} \cdot \bar{\chi}^{\prime \prime}\right)^{2}}{\left(2-\left\|\bar{\chi}^{\prime}\right\|^{2}\right)\left[\left\|\bar{\chi}^{\prime}\right\|^{2}\left(\overline{\mathbb{K}}^{e}-4 \overline{\mathbb{K}}^{f}\right)+8 \overline{\mathbb{K}}^{f}\right]} \mathrm{d} \overline{\mathrm{s}}+ \\
& +K^{m} B \int_{0}^{1} \frac{\overline{\mathbb{K}}^{m}}{2}\left(\left\|\bar{\chi}^{\prime}\right\|-1\right)^{2} \mathrm{~d} \overline{\mathrm{s}}
\end{aligned}
$$

where the symbol $\left.\left\langle<^{\bar{\top}}\right\rangle\right\rangle$ denotes differentiation with respect to the dimensionless abscissa $\bar{s}$.

\subsection{The Inextensibility Case}

Let us focus now on the inextensibility case. The homogenization procedure follows the same lines of the previous case. Indeed, keeping in mind (23) and (24), letting $\varepsilon \rightarrow 0$ in (21) yields the continuum-limit macro-model energy for the inextensibility case

$$
\begin{aligned}
& \int_{S}\left\{\mathbb{K}^{f}\left[\vartheta^{\prime 2}+\frac{\rho^{\prime 2}}{2-\rho^{2}}\right]+\frac{\mathbb{K}^{m}}{2}(\rho-1)^{2}\right\} \mathrm{ds}= \\
& =\int_{S}\left\{\mathbb{K}^{f}\left[\frac{\left(\chi_{\perp} \cdot \chi^{\prime \prime}\right)^{2}}{\left\|\chi^{\prime}\right\|^{4}}+\frac{\left(\chi \cdot \chi^{\prime \prime}\right)^{2}}{\left\|\chi^{\prime}\right\|^{2}\left(2-\left\|\chi^{\prime}\right\|^{2}\right)}\right]+\frac{\mathbb{K}^{m}}{2}\left(\left\|\chi^{\prime}\right\|-1\right)^{2}\right\} \mathrm{ds} .
\end{aligned}
$$

This result is consistent with the quasi-inextensibility case. Indeed, we could have found (29) also by letting $\mathbb{K}^{e} \rightarrow+\infty$ in (27). Let us remark that, also in this case, the homogenized continuum model, due to the richness of the mictrostructure, gives rise to a full second gradient theory. 


\subsubsection{Linearization}

An interesting connection can be traced with the existing literature on the formulation of 1D continuum homogenized model for microstructured media and, in particular, for pantographic ones. Indeed, this connection is traced by considering a linearization of the pantographic beam energy in the (complete) inextensibility case. We set $\chi(s)=\left(\begin{array}{l}s \\ 0\end{array}\right)+\eta \tilde{u}$, with $\tilde{u}$ independent of $\eta$, i.e. we linearize with respect to the displacement $u=\chi(s)-\left(\begin{array}{l}s \\ 0\end{array}\right)$, and $\mathbb{K}^{m}=0$. By means of simple algebra manipulations it is possible to derive the deformation energy in Eq. (5) (with $K^{+}=K^{-}$) of [10] (see also [21]):

$$
\int_{S} \mathbb{K}^{f}\left\|u^{\prime \prime}\right\|^{2} \mathrm{ds}
$$

We remark that in the linearized energy (30) the transverse displacement and the axial one decouple.

\section{Numerical Simulations}

In the sequel, $\mathbb{K}^{m}=0$ will be considered, which means that the standard quadratic additive elongation/shortening contribution to the deformation energy will be turned off. This is made in order to better highlight some non-standard features of the nearlyinextensible pantographic beam model. In this section we show numerical results for the quasi-inextensible and inextensible pantographic beam model and for the geometrically non-linear Euler model. We remind that these cases stand for $\mathbb{K}^{e}<+\infty$ and $\mathbb{K}^{e} \rightarrow+\infty$, respectively. Two benchmark tests are exploited in order to illustrate peculiar and non-standard features of the pantographic beam model. Convergence of the quasi-inextensible pantographic beam model to the completely inextensible one is shown, by means of a numerical example, as the macro-stiffness $\mathbb{K}^{e}$ related to elongation of the oblique springs approaches $+\infty$. This is due to the fact that, as it is clear from Eq. (25), if $\mathbb{K}^{e} \rightarrow+\infty$, then $\tilde{l}^{\alpha \beta} \rightarrow 0$. Of course, the same discussion and simulations can be made for the micro-model and this could be the subject of a further investigation. For the sake of self-consistence, we recall that the deformation energy of the geometrically non-linear Euler model employed in the following simulations is the following

$$
\begin{aligned}
& \int_{S}\left\{\frac{K^{e}}{2}\left(\left\|\chi^{\prime}\right\|-1\right)^{2}+\frac{K^{b}}{2}\left[\frac{\chi^{\prime \prime} \cdot \chi^{\prime \prime}}{\left\|\chi^{\prime}\right\|^{2}}-\left(\frac{\chi^{\prime} \cdot \chi^{\prime \prime}}{\left\|\chi^{\prime}\right\|^{2}}\right)^{2}\right]\right\} \mathrm{ds}= \\
& =\int_{S}\left\{\frac{K^{e}}{2}(\rho-1)^{2}+\frac{K^{b}}{2} \vartheta^{\prime 2}\right\} \mathrm{ds}
\end{aligned}
$$


and we notice that, while in the nearly-inextensible pantographic beam model both $\rho$ and $\vartheta$ can be enforced at the boundary, for the non-linear Euler model it can be done for $\vartheta$ only, as no spatial derivative of $\rho$ appears in the energy.

\subsection{Semi-circle Test}

We consider for both the nearly-inextensible pantographic beam model and the geometrically non-linear Euler beam model the reference domain to be the interval $[0,2 \pi]$. We enforce the following boundary conditions for both models

$$
\text { 1. } \chi(0)=\mathbf{0} ; \quad 2 . \chi(2 \pi)=2 \mathbf{e}_{1 ;} \quad 3 . \vartheta(0)=-\frac{\pi}{2} ; \quad 4 . \vartheta(2 \pi)=\frac{\pi}{2}
$$

and, for the nearly-inextensible pantographic beam model, we also have the following two additional constraints

$$
\text { 5. } \rho(0)=\rho_{0} ; \quad \text { 6. } \rho(2 \pi)=\rho_{0} \text {. }
$$

In Fig. 3 (up) the deformed shapes for the nearly-inextensible pantographic beam model and for the geometrically non-linear Euler beam model (GNEM) are shown for different values of $\rho_{0}$ reported in the legend. In Fig. 3 (down) the elongation $\rho-1$ for the nearly-inextensible pantographic beam model and for the geometrically non-linear Euler beam model (GNEM) is shown for different values of $\rho_{0}$ reported in the legend. It is remarkable that passing from $\rho_{0}>1$ to $\rho<1$ there is a change of concavity in the elongation for the pantographic beam model. In Fig. 4 (up) the deformed shapes for the nearly-inextensible pantographic beam model (blue) and for the inextensible pantographic beam model (green) with $\rho_{0}=1.4$ are compared. Of course, the area spanned by the quasi-inextensible pantographic beam includes that
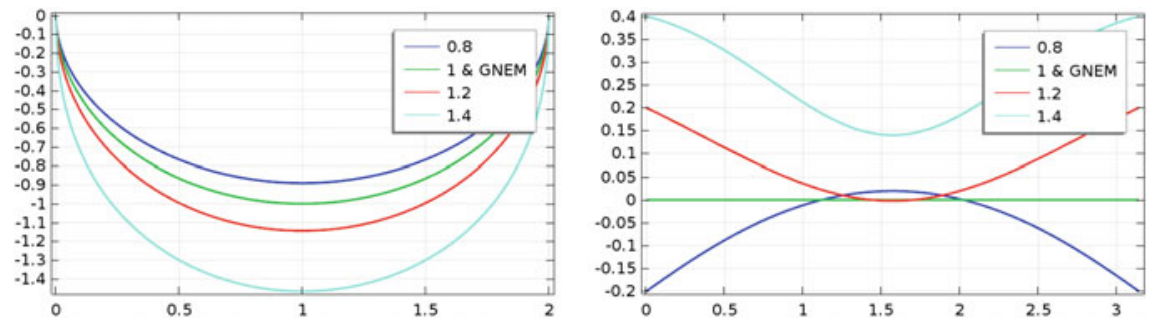

Fig. 3 Semi-circle test. Deformed shapes for the nearly-inextensible pantographic beam model and for the geometrically non-linear Euler beam model (GNEM). (left). Elongation $\rho-1$ versus the reference abscissafor the nearly-inextensible pantographic beam model and for the geometrically non-linear Euler beam model (GNEM) (right). Numbers in the legends stands for different dimensionless values of $\rho_{0}$ 

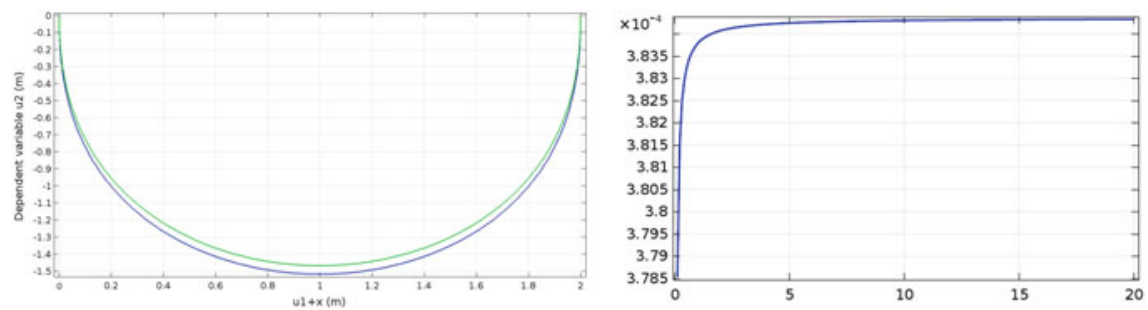

Fig. 4 Semi-circle test. Deformed shapes for the nearly-inextensible pantographic beam model (blue) and for the inextensible pantographic beam model (green) with $\rho_{0}=1.4$ (left). Energy of the nearly-inextensible pantographic beam model (ordinate) asymptotically tends to the energy of the inextensible pantographic beam model (asymptote) as $\mathbb{K}^{e}$ (abscissa) $\rightarrow+\infty$ (right)

of the (completely) inextensible one. In Fig. 4 (down) it is numerically shown that the energy of the nearly-inextensible pantographic beam model (ordinate) asymptotically tends to the energy of the inextensible pantographic beam model (asymptote) as $\mathbb{K}^{e}$ (abscissa) $\rightarrow+\infty$.

\subsection{Three-Point Test}

We consider for both the quasi-inextensible pantographic beam model and the geometrically non-linear Euler beam model the reference domain to be the interval $[0,2]$. We enforce the following boundary conditions for both models:

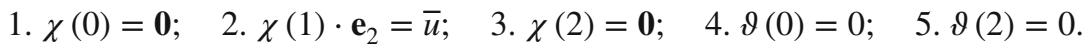

In Fig. 5 the deformed shapes for the nearly-inextensible pantographic beam model (red, light blue) and for the geometrically non-linear Euler beam model (blue, green) are shown for different values of $\bar{u}$ in the legend. Figure 6 shows, for different values of the parameter $\bar{u}$, the elongation $\rho-1$ versus the reference abscissa for the nearly-inextensible pantographic beam model. The parameter $\bar{u}$ is increasing from bottom to top. We observe that, as $\bar{u}$ increases, at some point, there is a concavity change in the elongation plot and, increasing further the parameter $\bar{u}$, curves start to intersect. This means that, for some points of the beam an increase of the prescribed displacement $\bar{u}$ implies a decrease in the elongation. Figure 7 shows the pulling force, i.e. Lagrange multiplier associated to the weak constraint $\chi(1) \cdot \mathbf{e}_{2}=\bar{u}$, changed of sign, applied at the midpoint in order to vertically displace it of an amount $\bar{u}$. In the nearly-inextensible pantographic beam model (blue) negative stiffness property, also known as elastic softening, is observed, while in the geometrically non-linear Euler beam model (green) elastic softening is not observed. Figure 8 shows the plot of $\tilde{l}^{1 D}$ versus reference abscissa for different values of $\bar{u}$ in the legend. Analogous plots hold for $\tilde{l}^{2 D}, \tilde{l}^{1 S}$ and $\tilde{l}^{2 S}$. 


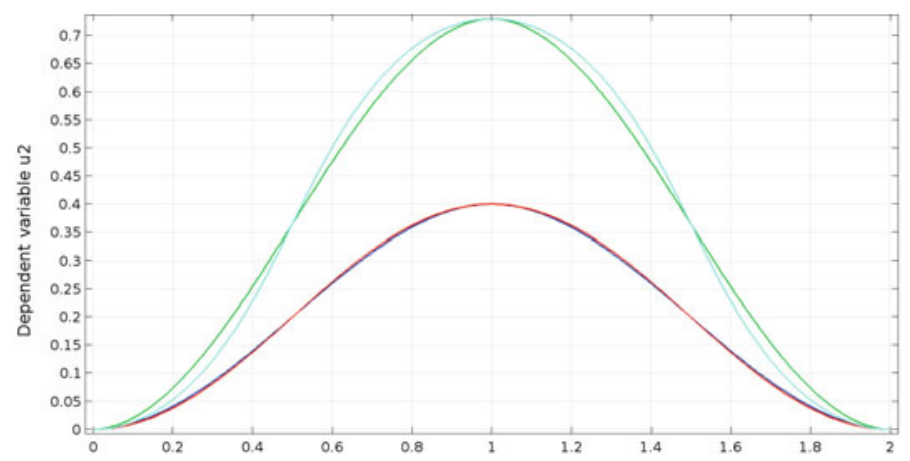

Fig. 5 Three-point test. Deformed shapes for the nearly-inextensible pantographic beam model (red, light blue) and for the geometrically non-linear Euler beam model (blue, green) for different values of $\bar{u}$ in the legend

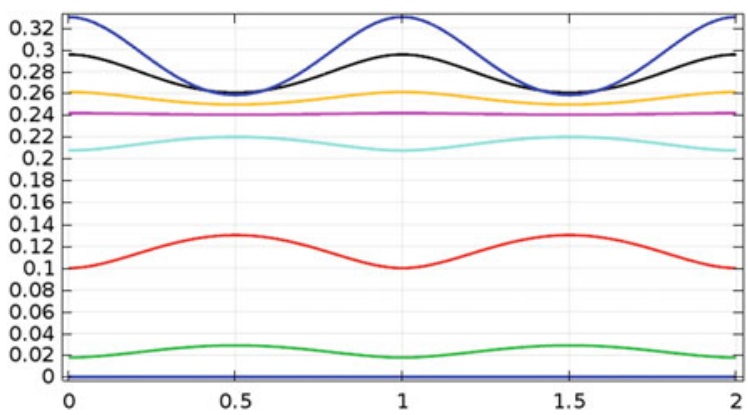

Fig. 6 Three-point test. Elongation $\rho-1$ versus the reference abscissa for the nearly-inextensible pantographic beam model. The parameter $\bar{u}$ is increasing from bottom to top. We observe that, While increasing $\bar{u}$, there is a concavity change at some point. Increasing further the parameter $\bar{u}$, curves start to intersect

\subsection{Modified Three-Point Test}

We consider for both the quasi-inextensible pantographic beam model and the geometrically non-linear Euler beam model the reference domain to be the interval $[0,2]$. We enforce the three-point test boundary conditions for both models
1. $\chi(0)=\mathbf{0}$;
2. $\chi(1) \cdot \mathbf{e}_{2}=\bar{u}$;
3. $\chi(2)=\mathbf{0}$;
4. $\vartheta(0)=0$;
5. $\vartheta(2)=0$

with the additional condition, at the midpoint $s=1$,

$$
\text { 6. } \rho(1) \simeq \sqrt{2} \text {. }
$$




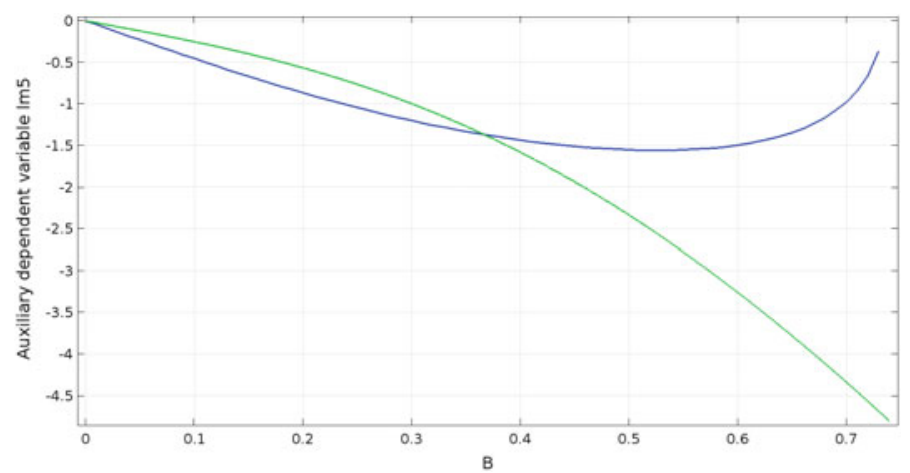

Fig. 7 Three-point test. Pulling force (i.e. Lagrange multiplier associated to the weak constraint $\chi(1) \cdot \mathbf{e}_{2}=\bar{u}$ ), changed of sign, applied at the midpoint in order to vertically displace it of an amount $\bar{u}$ (abscissa). In the nearly-inextensible pantographic beam model (blue) elastic softening is observed, while in the geometrically non-linear beam model (green) elastic softening is not observed

Fig. 8 Three-point test. Plot of $\tilde{l}^{1 D}$ versus reference abscissa for different values of $\bar{u}$ in the legend. Analogous plots hold for $\tilde{l}^{2 D}, \tilde{l}^{1 S}$ and $\tilde{l}^{2 S}$

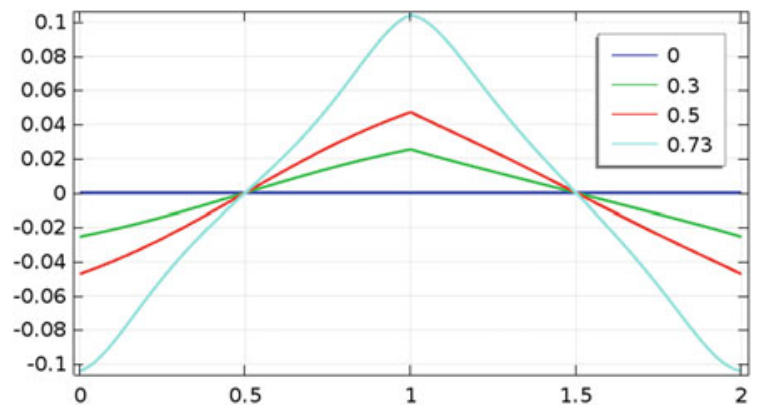

Fig. 9 shows the deformed configuration for the nearly-inextensible pantographic beam model, while in Fig. 10 the elongation $\rho-1$ versus the reference abscissa for the nearly-inextensible pantographic beam model is shown.

\section{Euler-Lagrange Equations}

Let us consider an internal (potential) unit line energy density of the form

$$
W=\frac{G(\rho)}{2} \rho^{\prime 2}+\frac{F(\rho)}{2} \vartheta^{\prime 2}+H(\rho)
$$

with $\rho$ and $\vartheta$ such that $\chi^{\prime}=\rho\left(\cos \vartheta \mathbf{e}_{1}+\sin \vartheta \mathbf{e}_{2}\right)=\rho \mathbf{e}(\vartheta)$, and $G, F, H$ functions from $\mathbb{R}^{+} \supseteq A$ to $\mathbb{R}^{+}$. We recall that $\rho^{\prime}=\frac{\chi^{\prime \prime} \cdot \chi^{\prime}}{\left\|\chi^{\prime}\right\|}, \vartheta^{\prime}=\frac{\chi^{\prime \prime} \cdot \chi_{\perp}^{\prime}}{\left\|\chi^{\prime}\right\|^{2}}$. We remark that, when 


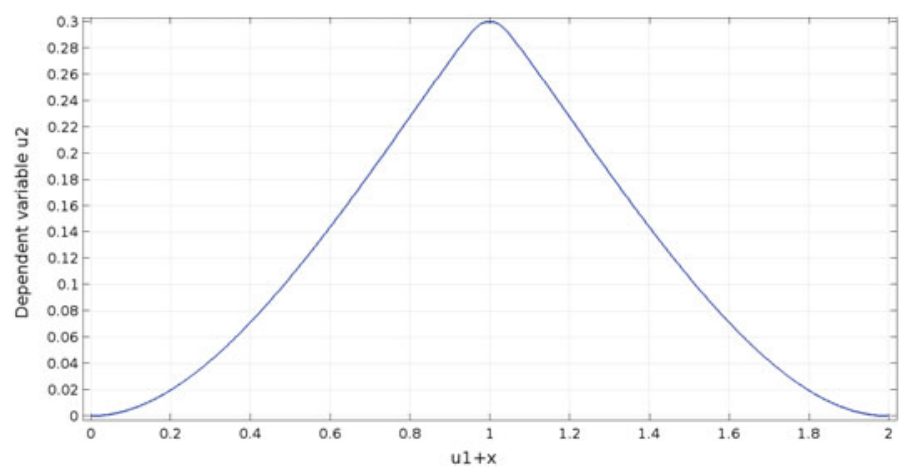

Fig. 9 Modified three-point test. Deformed configuration for the nearly-inextensible pantographic beam model

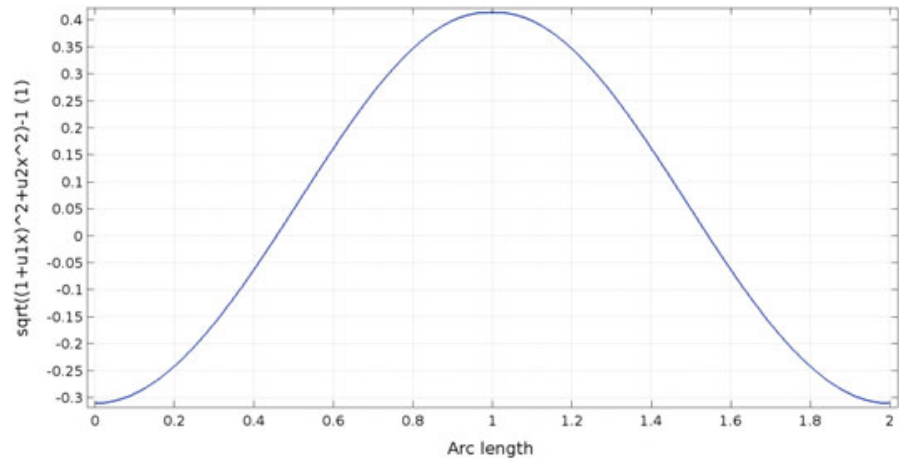

Fig. 10 Modified three-point test. Elongation $\rho-1$ versus reference abscissa for the nearlyinextensible pantographic beam model

the specialization

$$
\left\{\begin{array}{l}
G(\rho)=0 \\
F(\rho)=K^{b} \geq 0 \\
H(\rho)=\frac{K^{e}}{2}(\rho-1)^{2}, K^{e} \geq 0
\end{array}\right.
$$

of (31) is considered, we find the geometrically nonlinear Euler model while, when the specialization

$$
\left\{\begin{array}{l}
G(\rho)=\mathbb{K}^{e} \mathbb{K}^{f} \frac{\rho^{2}}{\left(2-\rho^{2}\right)\left[\rho^{2}\left(\mathbb{K}^{e}-4 \mathbb{K}^{f}\right)+8 \mathbb{K}^{f}\right]}, \mathbb{K}^{e} \geq 0, \mathbb{K}^{f} \geq 0 \\
F(\rho)=\mathbb{K}^{e} \mathbb{K}^{f} \frac{\left(\rho^{2}-2\right)}{\rho^{2}\left(\mathbb{K}^{e}-4 \mathbb{K}^{f}\right)-2 \mathbb{K}^{e}}, \mathbb{K}^{e} \geq 0, \mathbb{K}^{f} \geq 0 \\
H(\rho)=\frac{\mathbb{K}^{m}}{2}(\rho-1)^{2}, \mathbb{K}^{m}>0
\end{array}\right.
$$


of (31) is considered, we find the nearly-inextensible pantographic beam model. We now consider the functional

$$
\begin{aligned}
& \mathcal{E}(\rho(\cdot), \vartheta(\cdot), \chi(\cdot), \Lambda(\cdot))= \\
& =\int_{S}\left\{W-b^{e x t} \cdot \chi-\mu^{e x t} \cdot \vartheta+\Lambda \cdot\left[\chi^{\prime}-\rho \mathbf{e}(\vartheta)\right]\right\} \mathrm{ds}+ \\
& -\sum_{s=0, L} R^{e x t}(s) \cdot \chi(s)-\sum_{s=0, L} M^{e x t}(s) \cdot \vartheta(s),
\end{aligned}
$$

where $\int_{S} b^{e x t} \cdot \chi+\mu^{e x t} \cdot \vartheta \mathrm{ds}+\sum_{s=0, L} R^{e x t}(s) \cdot \chi(s)+\sum_{s=0, L} M^{\text {ext }}(s) \cdot \vartheta(s)$ is the work done by external distributed and concentrated forces and couples. The first variation of the functional $\mathcal{E}$ in (34) is

$$
\begin{aligned}
& \delta \mathcal{E}(\rho(\cdot), \vartheta(\cdot), \chi(\cdot), \Lambda(\cdot), \delta \rho(\cdot), \delta \vartheta(\cdot), \delta \chi(\cdot), \delta \Lambda(\cdot))= \\
& =\int_{S}\left\{\frac{1}{2} \frac{\partial G}{\partial \rho}(\rho) \rho^{\prime 2}+\frac{1}{2} \frac{\partial F}{\partial \rho}(\rho) \vartheta^{\prime 2}+\frac{\partial H}{\partial \rho}(\rho)-\Lambda \cdot \mathbf{e}(\vartheta)-\left[G(\rho) \rho^{\prime}\right]^{\prime}\right\} \delta \rho \mathrm{ds}+ \\
& +\int_{S}\left\{-\left[F(\rho) \vartheta^{\prime}\right]^{\prime}-\mu-\Lambda \times \chi^{\prime}\right\} \delta \vartheta+\left(-b-\Lambda^{\prime}\right) \cdot \delta \chi+\left[\chi^{\prime}-\rho \mathbf{e}(\vartheta)\right] \cdot \delta \Lambda \mathrm{ds}+ \\
& +\left[G(\rho) \rho^{\prime} \delta \rho\right]_{0}^{L}+\left\{\left[F(\rho) \vartheta^{\prime}-M^{e x t}\right] \delta \vartheta\right\}_{0}^{L}+\left[\left(\Lambda-R^{e x t}\right) \cdot \delta \chi\right]_{0}^{L}= \\
& =\int_{S}\left\{\frac{1}{2} \frac{\partial F}{\partial \rho}(\rho) \vartheta^{\prime 2}+\frac{\partial H}{\partial \rho}(\rho)-\frac{1}{2} \frac{\partial G}{\partial \rho}(\rho) \rho^{\prime 2}-\Lambda \cdot \mathbf{e}(\vartheta)-G(\rho) \rho^{\prime \prime}\right\} \delta \rho \mathrm{ds}+ \\
& +\int_{S}\left\{-\left[F(\rho) \vartheta^{\prime}\right]^{\prime}-\mu-\Lambda \times \chi^{\prime}\right\} \delta \vartheta+\left(-b-\Lambda^{\prime}\right) \cdot \delta \chi+\left[\chi^{\prime}-\rho \mathbf{e}(\vartheta)\right] \cdot \delta \Lambda \mathrm{ds}+ \\
& +\left[G(\rho) \rho^{\prime} \delta \rho\right]_{0}^{L}+\left\{\left[F(\rho) \vartheta^{\prime}-M^{e x t}\right] \delta \vartheta\right\}_{0}^{L}+\left[\left(\Lambda-R^{e x t}\right) \cdot \delta \chi\right]_{0}^{L} .
\end{aligned}
$$

By applying the Fundamental Lemma of Calculus of Variations, we find the EulerLagrange equations:
1. $\frac{1}{2} \frac{\partial G}{\partial \rho}(\rho) \rho^{\prime 2}+\frac{1}{2} \frac{\partial F}{\partial \rho}(\rho) \vartheta^{\prime 2}+\frac{\partial H}{\partial \rho}(\rho)-\Lambda \cdot \mathbf{e}(\vartheta)-\left[G(\rho) \rho^{\prime}\right]^{\prime}=0$
2. $\left[F(\rho) \vartheta^{\prime}\right]^{\prime}+\mu+\Lambda \times \chi^{\prime}=0$

3. $b+\Lambda^{\prime}=0$

4. $\chi^{\prime}-\rho \mathbf{e}(\vartheta)=0$ 
and the corresponding boundary conditions:

$$
\begin{aligned}
& G(\rho(0))=0 \vee \rho^{\prime}(0)=0 \vee \text { Dirichlet Conditions on } \rho \text { in } s=0 \\
& G(\rho(L))=0 \vee \rho^{\prime}(L)=0 \vee \text { Dirichlet Conditions on } \rho \text { in } s=L
\end{aligned}
$$

$F(\rho(0)) \vartheta^{\prime}(0)-M^{e x t}(0)=0 \vee$ Dirichlet Conditions on $\vartheta$ in $s=0$

$F(\rho(L)) \vartheta^{\prime}(L)-M^{e x t}(L)=0 \vee$ Dirichlet Conditions on $\vartheta$ in $s=L$

$$
\begin{aligned}
& \Lambda(0)-R^{e x t}(0)=0 \vee \text { Dirichlet Conditions on } \chi \text { in } s=0 \\
& \Lambda(L)-R^{e x t}(L)=0 \vee \text { Dirichlet Conditions on } \chi \text { in } s=L .
\end{aligned}
$$

We now analyze the two specializations (32) and (33) of (31). Let us first analyze (32). The Euler-Lagrange equations reduce to

$$
\begin{aligned}
& \text { 1. } K^{e}(\rho-1)-\Lambda \cdot \mathbf{e}(\vartheta)=0 \\
& \text { 2. }\left[K^{b} \vartheta^{\prime}\right]^{\prime}+\mu+\Lambda \times \chi^{\prime}=0 \\
& \text { 3. } b+\Lambda^{\prime}=0 \\
& \text { 4. } \chi^{\prime}-\rho \mathbf{e}(\vartheta)=0,
\end{aligned}
$$

complemented with the following boundary conditions

$$
\begin{aligned}
& K^{b} \vartheta^{\prime}(0)-M^{e x t}(0)=0 \vee \text { Dirichlet Conditions on } \vartheta \text { in } s=0 \\
& K^{b} \vartheta^{\prime}(L)-M^{e x t}(L)=0 \vee \text { Dirichlet Conditions on } \vartheta \text { in } s=L
\end{aligned}
$$

$$
\begin{aligned}
& \Lambda(0)-R^{e x t}(0)=0 \vee \text { Dirichlet Conditions on } \chi \text { in } s=0 \\
& \Lambda(L)-R^{\text {ext }}(L)=0 \vee \text { Dirichlet Conditions on } \chi \text { in } s=L .
\end{aligned}
$$

We notice that (35) 1 is an algebraic equation in $\rho$, and it gives:

$$
\rho=1+\frac{\Lambda \cdot \mathbf{e}(\vartheta)}{K^{e}} .
$$


The direct integration of Eq. (35) $)_{3}$ gives:

$$
\Lambda=\int_{0}^{s} b \mathrm{ds}+R^{e x t}(0),
$$

while, plugging (38) in (37) yields

$$
\rho=1+\frac{\left[\int_{0}^{s} b \mathrm{ds}+R^{e x t}(0)\right] \cdot \mathbf{e}(\vartheta)}{K^{e}}
$$

and plugging (39) and (38) in (35) 2 yields

$$
\left[K^{b} \vartheta^{\prime}\right]^{\prime}+\mu+\Lambda \times\left(1+\frac{\Lambda \cdot \mathbf{e}(\vartheta)}{K^{e}}\right) \mathbf{e}(\vartheta)=0,
$$

which is a 2 nd order O.D.E. in the unknown $\vartheta$. This O.D.E. is complemented with boundary conditions (36) $)_{1,2}$. Finally, one recovers $\chi$ by integrating $(35)_{4}$ and by using the Dirichlet boundary conditions on $\chi$. Let us turn to the study of the specialization (33) of the unit line potential energy density in Eq. (31). By explicitly computing the partial derivatives of the functions $F, G, H$ with respect to $\rho$, we get the following Euler-Lagrange equations for the nearly-inextensible pantographic beam model:

$$
\begin{aligned}
& \text { 1. } \frac{\mathbb{K}^{e} \mathbb{K}^{f}\left[16 \mathbb{K}^{f} \rho+\left(\mathbb{K}^{e}-4 \mathbb{K}^{f}\right) \rho^{5}\right]}{\left(\rho^{2}-2\right)^{2}\left[\left(\mathbb{K}^{e}-4 \mathbb{\mathbb { K } ^ { f }}\right) \rho^{2}+8 \mathbb{K}^{f}\right]^{2}} \rho^{\prime 2}-\frac{16 \mathbb{K}^{e}\left(\mathbb{K}^{f}\right)^{2} \rho}{\left.\left[\left(\mathbb{K}^{e}-4 \mathbb{K}^{f}\right) \rho^{2}-2 \mathbb{K}^{e}\right)\right]^{2}} \vartheta^{\prime 2}+\mathbb{K}^{m}(\rho-1)-\Lambda \cdot \mathbf{e}(\vartheta)+ \\
&-\left[\mathbb{K}^{e} \mathbb{K}^{f} \frac{\rho^{2} \rho^{\prime}}{\left(2-\rho^{2}\right)\left[\rho^{2}\left(\mathbb{K}^{e}-4 \mathbb{K}^{f}\right)+8 \mathbb{K}^{f}\right]}\right]^{\prime}=0
\end{aligned}
$$

2. $\left[\mathbb{K}^{e} \mathbb{K}^{f} \frac{\left(\rho^{2}-2\right)}{\rho^{2}\left(\mathbb{K}^{e}-4 \mathbb{K}^{f}\right)-2 \mathbb{K}^{e}} \vartheta^{\prime}\right]^{\prime}+\mu+\Lambda \times \chi^{\prime}=0$

3. $b+\Lambda^{\prime}=0$

4. $\chi^{\prime}-\rho \mathbf{e}(\vartheta)=0$,

complemented with the following boundary conditions:

$\rho^{\prime}(0)=0 \vee$ Dirichlet Conditions on $\rho$ in $s=0$

$\rho^{\prime}(L)=0 \vee$ Dirichlet Conditions on $\rho$ in $s=L$

$\mathbb{K}^{e} \mathbb{K}^{f} \frac{\left(\rho^{2}(0)-2\right)}{\rho^{2}(0)\left(\mathbb{K}^{e}-4 \mathbb{K}^{f}\right)-2 \mathbb{K}^{e}} \vartheta^{\prime}(0)-M^{e x t}(0)=0 \vee$ Dirichlet Conditions on $\vartheta$ in $s=0$

$\mathbb{K}^{e} \mathbb{K}^{f} \frac{\left(\rho^{2}(L)-2\right)}{\rho^{2}(L)\left(\mathbb{K}^{e}-4 \mathbb{K}^{f}\right)-2 \mathbb{K}^{e}} \vartheta^{\prime}(L)-M^{e x t}(L)=0 \vee$ Dirichlet Conditions on $\vartheta$ in $s=L$

$$
\begin{aligned}
& \Lambda(0)-R^{e x t}(0)=0 \vee \text { Dirichlet Conditions on } \chi \text { in } s=0 \\
& \Lambda(L)-R^{e x t}(L)=0 \vee \text { Dirichlet Conditions on } \chi \text { in } s=L .
\end{aligned}
$$


For the (completely) inextensible pantographic beam model, from Eq. (29) we have that:

$$
\begin{aligned}
& G(\rho)=\frac{2 \mathbb{K}^{f}}{2-\rho^{2}} \\
& F(\rho)=2 \mathbb{K}^{f} \\
& H(\rho)=\mathbb{K}^{m}(\rho-1)^{2} .
\end{aligned}
$$

Therefore, Euler-Lagrange equations for the inextensible pantographic beam model recast as

$$
\text { 1. } \frac{2 \rho \mathbb{K}^{f}}{\left(2-\rho^{2}\right)^{2}} \rho^{\prime 2}+2 \mathbb{K}^{m}(\rho-1)-\Lambda \cdot \mathbf{e}(\vartheta)-\left[\frac{2 \mathbb{K}^{f}}{2-\rho^{2}} \rho^{\prime}\right]=0^{\prime}
$$

2. $\mu+\Lambda \times \chi^{\prime}=0$

3. $b+\Lambda^{\prime}=0$

4. $\chi^{\prime}-\rho \mathbf{e}(\vartheta)=0$,

complemented with the following boundary conditions:

$$
\begin{aligned}
& \rho^{\prime}(0)=0 \vee \text { Dirichlet Conditions on } \rho \text { in } s=0 \\
& \rho^{\prime}(L)=0 \vee \text { Dirichlet Conditions on } \rho \text { in } s=L \\
& 2 \mathbb{K}^{f} \vartheta^{\prime}(0)-M^{\text {ext }}(0)=0 \vee \text { Dirichlet Conditions on } \vartheta \text { in } s=0 \\
& 2 \mathbb{K}^{f} \vartheta^{\prime}(L)-M^{\text {ext }}(L)=0 \vee \text { Dirichlet Conditions on } \vartheta \text { in } s=L \\
& \Lambda(0)-R^{\text {ext }}(0)=0 \vee \text { Dirichlet Conditions on } \chi \text { in } s=0 \\
& \Lambda(L)-R^{\text {ext }}(L)=0 \vee \text { Dirichlet Conditions on } \chi \text { in } s=L .
\end{aligned}
$$

We believe that the use of the techniques employed in [74], which have been exploited to analytically determine remarkable properties of the extensible Euler beam model in finite deformation regime, can be fruitfully applied to the examined pantographic beam model. Also the numerical exploitation of the Euler-Lagrange equations through, e.g., a shooting method, could be useful to explore the set of solutions. This might be of help in shedding light on the mathematical properties of the quasi-inextensible and inextensible pantographic beam models.

\section{Conclusions}

In this work, we have modeled and analyzed a pantographic microstructure giving rise, after a homogenization procedure, to a $1 D$ continuum model for a beam which 
exhibits many interesting non-standard features. We have provided a characterization of the kinematics of the proposed discrete Hencky-type spring model which resembles the geometrical arrangement of a pantographic strip. Once the kinematics of the micro-model has been discussed, we have given a mechanical characterization of the micro-system, by specifying the general form of the deformation energy. Subsequently, we postulated an asymptotic expansion for one of the microscopic descriptors, i.e. the elongation of the oblique springs. Besides, by choosing the value of the first-order term of this expansion, we considered the so-called quasi-inextensibility case, which corresponds to the quasi-inextensibility of the oblique springs. Successively, following the Piola's ansatz, we introduced the so-called kinematical maps, i.e. some fields in the macroscopic model that uniquely determine the microscopic kinematical descriptors. By means of these kinematical maps, namely the placement function $\chi$ and the elongation of the oblique springs $\tilde{l}^{\alpha \beta}$, we have written the micromodel energy for the quasi-inextensibility case in terms of macroscopic descriptors. The resulting micro-model energy contains already a contribution from the second gradient of $\chi(s)$ and it provides an upper bound $\left\|\chi^{\prime}\right\|<\sqrt{2}$, even if no kinematic restrictions directly affect $\left\|\chi^{\prime}\right\|$. Successively, we have considered the case of (completely) inextensible oblique springs by means of two equivalent procedures. We also verified that, as it is obvious, requiring the kinematic descriptor $\tilde{l}^{\alpha \beta}$, standing for the elongation of the oblique springs, to vanish implies that the quasi-inextensible pantographic beam model specializes to the (completely) inextensible pantographic beam model. In order to give a better insight into the peculiar properties of this problem, we also derived "ab imis fundamentis" the micro-model energy of the inextensible pantographic beam model, whose kinematics is characterized by the positions $p_{i}$ only, as a function of the macroscopic kinematical descriptor $\chi$ evaluated in $s_{i}$ 's. Following a procedure analogue to the quasi-inextensibility case, we defined the micro-model energy for the inextensibility case and, once the kinematical maps was introduced by means of the Piola's ansatz, we have written it in terms of these macroscopic descriptors. Then, we have imposed the so-called internal connection constraint, which ensures that, in the deformed configuration, the upper-left spring of the $i$-th cell is hinge-joint with the upper-right spring of the $(i-1)$-th cell, and the lower-left spring of the $i$-th cell is hinge-joint with lower-right spring of the $(i-1)$-th cell. After that, we have performed a heuristic homogenization procedure in order to derive a $1 D$ continuum model, also referred to as the macro-model and characterized by its deformation energy, associated to the aforementioned microstructure in the quasi-inextensibility and inextensibility cases. The preliminary step to perform the homogenization procedure has been to define scale-invariant quantities, whose role is to keep track of the asymptotic behaviour of the stiffnesses of the micro-model springs. We first performed the homogenization procedure for the quasi-inextensibility case by computing the limit $\varepsilon \rightarrow 0$ in the expression of the micro-model energy written in terms of macroscopic kinematical descriptors. The resulting continuum-limit macro-model energy describes a $1 D$ pantographic beam under the hypothesis of quasi-inextensible oblique micro-springs. The macroscopic beam, when $\mathbb{K}^{m}=0, \tilde{l}^{\alpha \beta}=0$, and $\chi(s)=C s \boldsymbol{e}_{1}$, undergoes a floppy mode. This is 
a key feature of the micro-model energy which was preserved by the homogenization procedure that we have carried out. Moreover, we observed that not only this homogenized model can be classified as a second gradient theory, but we noticed that the full second gradient $\chi^{\prime \prime}$ of $\chi$ contributes to the strain energy. Indeed, beyond the usual term $\left(\chi_{\perp}^{\prime} \cdot \chi^{\prime \prime}\right)$ related to the Lagrangian curvature, also the term $\left(\chi^{\prime} \cdot \chi^{\prime \prime}\right)$, deriving from the presence of the oblique springs, appears. Finally, we remarked that, in the limit $\left\|\chi^{\prime}\right\| \rightarrow \sqrt{2}$, the model exhibits a so-called phase transition, i.e. it locally degenerates into the model of an uniformly extensible cable, notwithstanding that $\sqrt{2}$ is an upper bound for $\rho$. Subsequently, we have performed the homogenization procedure also for the (complete) inextensibility case. The homogenization procedure has followed the same lines of the quasi-inextensibility case and it yields a continuum-limit macro-model energy consistent with the quasi-inextensibility case. An interesting connection with the existing literature on $1 D$ continuum homogenized models for microstructured media, and in particular for pantographic ones, has been traced by considering the linearization of the pantographic beam energy in the (complete) inextensibility case. Furthermore, it has to be remarked that, of course, what has been presented in this paper is not the only possible homogenization technique. Indeed, many other procedures, like coarse-graining, hydrodynamical limits [76-79] for many-particle systems, and computational homogenization [80-82], are being employed in literature, and they deserve to be better understood. The numerical solution of the homogenised continuum model has been addressed by means of the finite element method in three exemplary cases, trying to highlight the main differences with the classical finite deformation Euler beam model. In particular, in order to better highlight some non-standard features of the nearly-inextensible pantographic beam model, we have considered as vanishing the standard quadratic additive elongation/shortening contribution to the deformation energy. These benchmark tests were exploited in order to illustrate some peculiar features of the pantographic beam model and the convergence of the quasi-inextensible pantographic beam model to the completely inextensible one. In particular, we have performed for the nearly-inextensible pantographic beam model and for the geometrically non-linear Euler model what has been referred to as the semi-circle test, the three-point test and the modified threepoint test. The weak form deriving from the stationarity of the energy functional has been projected on the basis of Hermite cubic interpolants. Further improvements of this approach could include the use of isogeometric analysis, which has found wide application for beam elements [83-91]. Indeed, spline functions employed in that approach allow to ensure higher continuity between elements and other many desirable properties. Finally, through a standard variational procedure, we have provided the explicit form of the Euler-Lagrange equations and of the corresponding boundary conditions for a general potential energy density functional which includes as particular cases the quasi-inextensible pantographic beam model and the Euler beam model. Moreover, we have given the explicit form of the Euler-Lagrange equations and of the boundary conditions for the quasi-inextensible and completely inextensible pantographic beam model. It is conceivable that pantographic beams can be used as building blocks for more complex double scale materials. 
1. Euler, L., Carathéodory, C.: Methodus Inveniendi Lineas Curvas Maximi Minimive Proprietate Gaudentes Sive Solutio Problematis Isoperimetrici Latissimo Sensu Accepti, vol. 1. Springer Science \& Business Media (1952)

2. Antman, S.S.: Nonlinear Problems of Elasticity. Mathematical Sciences, vol. 107. Springer, Berlin, New York (1995)

3. Placidi, L., Barchiesi, E., Battista, A.: An inverse method to get further analytical solutions for a class of metamaterials aimed to validate numerical integrations. In: Mathematical Modelling in Solid Mechanics, pp. 193-210. Springer (2017)

4. Murat, F., Sili, A.: Comportement asymptotique des solutions du système de l'élasticité linéarisée anisotrope hétérogène dans des cylindres minces. Comptes Rendus de l'Académie des Sciences-Series I-Mathematics 328(2), 179-184 (1999)

5. Mora, M.G., Müller, S.: A nonlinear model for inextensible rods as a low energy $\gamma$-limit of three-dimensional nonlinear elasticity. Annales de l'IHP Analyse non linéaire 21, 271-293 (2004)

6. Jamal, R., Sanchez-Palencia, E.: Théorie asymptotique des tiges courbes anisotropes. Comptes rendus de l'Académie des sciences. Série 1, Mathématique 322(11), 1099-1106 (1996)

7. Pideri, C., Seppecher, P.: Asymptotics of a non-planar rod in non-linear elasticity. Asymptot. Anal. 48(1, 2), 33-54 (2006)

8. Allaire, G.: Homogenization and two-scale convergence. SIAM J. Math. Anal. 23(6), 1482 1518 (1992)

9. Bensoussan, A., Lions, J.-L., Papanicolaou, G.: Asymptotic Analysis for Periodic Structures, vol. 5. North-Holland Publishing Company Amsterdam (1978)

10. Alibert, J.-J., Seppecher, P., dell'Isola, F.: Truss modular beams with deformation energy depending on higher displacement gradients. Math. Mech. Solids 8(1), 51-73 (2003)

11. Carcaterra, A., dell'Isola, F., Esposito, R., Pulvirenti, M.: Macroscopic description of microscopically strongly inhomogeneous systems: a mathematical basis for the synthesis of higher gradients metamaterials. Arch. Ration. Mech. Anal. 218(3), 1239-1262 (2015)

12. Abali, B.E., Müller, W.H., dell'Isola, F.: Theory and computation of higher gradient elasticity theories based on action principles. Arch. Appl. Mech. 1-16 (2017)

13. Pietraszkiewicz, W., Eremeyev, V.: On natural strain measures of the non-linear micropolar continuum. Int. J. Solids Struct. 46(3), 774-787 (2009)

14. Altenbach, H., Eremeyev, V.: On the linear theory of micropolar plates. ZAMM-Journal of Applied Mathematics and Mechanics/Zeitschrift für Angewandte Mathematik und Mechanik 89(4), 242-256 (2009)

15. dell'Isola, F., Della Corte, A., Giorgio, I.: Higher-gradient continua: the legacy of piola, mindlin, sedov and toupin and some future research perspectives. Math. Mech. Solids (2016). https://doi.org/10.1177/1081286515616034

16. dell Isola, F., Seppecher, P., Della Corte, A.: The postulations á la d alembert and á la cauchy for higher gradient continuum theories are equivalent: a review of existing results. In: Proceedings of the Royal Society A, vol. 471, p. 20150415. The Royal Society (2015)

17. dell'Isola, F., Giorgio, I., Andreaus, U.: Elastic pantographic 2D lattices: a numerical analysis on static response and wave propagation. Proc. Est. Acad. Sci. 64, 219-225 (2015)

18. Reiher, J.C., Giorgio, I., Bertram, A.: Finite-element analysis of polyhedra under point and line forces in second-strain gradient elasticity. J. Eng. Mech. 143(2), 04016112 (2016)

19. Boutin, C., Giorgio, I., Placidi, L., et al.: Linear pantographic sheets: asymptotic micro-macro models identification. Math. Mech. Complex Syst. 5(2), 127-162 (2017)

20. dell'Isola, F., Cuomo, M., Greco, L., Della Corte, A.: Bias extension test for pantographic sheets: numerical simulations based on second gradient shear energies. J. Eng. Math. 1-31 (2016)

21. Seppecher, P., Alibert, J.-J., dell'Isola, F.: Linear elastic trusses leading to continua with exotic mechanical interactions. In: Journal of Physics: Conference Series, vol. 319, p. 012018. IOP Publishing (2011) 
22. Cuomo, M., dell'Isola, F., Greco, L., Rizzi, N.L.: First versus second gradient energies for planar sheets with two families of inextensible fibres: investigation on deformation boundary layers, discontinuities and geometrical instabilities. Eng. Compos. Part B (2016)

23. dell'Isola, F., Madeo, A., Seppecher, P.: Cauchy tetrahedron argument applied to higher contact interactions. Arch. Ration. Mech. Anal. 219(3), 1305-1341 (2016)

24. Placidi, L., Greco, L., Bucci, S., Turco, E., Rizzi, N.L.: A second gradient formulation for a 2D fabric sheet with inextensible fibres. Zeitschrift für angewandte Mathematik und Physik, 67(5)(114) (2016)

25. Enakoutsa, K., Della Corte, A., Giorgio, I.: A model for elastic flexoelectric materials including strain gradient effects. Math. Mech. Solids (2015). https://doi.org/10.1177/1081286515588638

26. Placidi, L., Andreaus, U., Giorgio, I.: Identification of two-dimensional pantographic structure via a linear d4 orthotropic second gradient elastic model. J. Eng. Math. 1-21 (2016)

27. Giorgio, I., Andreaus, U., Lekszycki, T., Della Corte, A.: The influence of different geometries of matrix/scaffold on the remodeling process of a bone and bioresorbable material mixture with voids. Math. Mech. Solids (2015). https://doi.org/10.1177/1081286515616052

28. Andreaus, U., Giorgio, I., Lekszycki, T.: A 2D continuum model of a mixture of bone tissue and bio-resorbable material for simulating mass density redistribution under load slowly variable in time. Zeitschrift für Angewandte Mathematik und Mechanik 13, 7 (2013)

29. Andreaus, U., Giorgio, I., Madeo, A.: Modeling of the interaction between bone tissue and resorbable biomaterial as linear elastic materials with voids. Zeitschrift für angewandte Mathematik und Physik 66(1), pp. 209-237 (2014)

30. Andreaus, U., Placidi, L., Rega, G.: Numerical simulation of the soft contact dynamics of an impacting bilinear oscillator. Commun. Nonlinear Sci. Numer. Simul. 15(9), 2603-2616 (2010)

31. Giorgio, I., Corte, A.Della: Dynamics of 1D nonlinear pantographic continua. Nonlinear Dyn. 88(1), 21-31 (2017)

32. Turco, E., Golaszewski, M., Giorgio, I., D’Annibale, F.: Pantographic lattices with nonorthogonal fibres: experiments and their numerical simulations. Compos. Part B: Eng. 118, 1-14 (2017)

33. Placidi, L., Andreaus, U., Della Corte, A., Lekszycki, T.: Gedanken experiments for the determination of two-dimensional linear second gradient elasticity coefficients. Zeitschrift für angewandte Mathematik und Physik 66(6), 3699-3725 (2015)

34. dell'Isola, F., Della Corte, A., Greco, L., Luongo, A.: Plane bias extension test for a continuum with two inextensible families of fibers: a variational treatment with lagrange multipliers and a perturbation solution. Int. J. Solids Struct. (2015)

35. Abali, B.E., Müller, W.H., Eremeyev, V.A.: Strain gradient elasticity with geometric nonlinearities and its computational evaluation. Mech. Adv. Mater. Mod. Process. 1(1), 4 (2015)

36. Auffray, N., dell'Isola, F., Eremeyev, V., Madeo, A., Rosi, G.: Analytical continuum mechanics à la Hamilton-Piola least action principle for second gradient continua and capillary fluids. Math. Mech. Solids 20(4), 375-417 (2015)

37. Yang, Y., Misra, A.: Micromechanics based second gradient continuum theory for shear band modeling in cohesive granular materials following damage elasticity. Int. J. Solids Struct. 49(18), 2500-2514 (2012)

38. Misra, A., Poorsolhjouy, P.: Granular micromechanics model for damage and plasticity of cementitious materials based upon thermomechanics. Math. Mech. Solids (2015). https://doi. org/10.1177/1081286515576821

39. Misra, A.1., Singh, V.: Thermomechanics-based nonlinear rate-dependent coupled damageplasticity granular micromechanics model. Contin. Mech. Thermodyn. 27(4-5), 787 (2015)

40. Della Corte, A., Battista, A., dell'Isola, F.: Referential description of the evolution of a 2D swarm of robots interacting with the closer neighbors. Int. J. Non-Linear Mech. 80, 209-220 (2016)

41. Del Vescovo, D., Giorgio, I.: Dynamic problems for metamaterials: review of existing models and ideas for further research. Int. J. Eng. Sci. 80, 153-172 (2014) 
42. Rinaldi, A., Placidi, L.: A microscale second gradient approximation of the damage parameter of quasi-brittle heterogeneous lattices. ZAMM-Journal of Applied Mathematics and Mechanics/Zeitschrift für Angewandte Mathematik und Mechanik 94(10), 862-877 (2014)

43. Placidi, L.: A variational approach for a nonlinear 1-dimensional second gradient continuum damage model. Contin. Mech. Thermodyn. 27(4-5), 623 (2015)

44. Madeo, A., Placidi, L., Rosi, G.: Towards the design of metamaterials with enhanced damage sensitivity: second gradient porous materials. Res. Nondestruct. Eval. 25(2), 99-124 (2014)

45. Misra, A.: Effect of asperity damage on shear behavior of single fracture. Eng. Fract. Mech. 69(17), 1997-2014 (2002)

46. Misra, A., Singh, V.: Micromechanical model for viscoelastic materials undergoing damage. Contin. Mech. Thermodyn. 1-16 (2013)

47. Yang, Y., Misra, A.: Higher-order stress-strain theory for damage modeling implemented in an element-free galerkin formulation. CMES-Comput. Model. Eng. Sci. 64(1), 1-36 (2010)

48. Madeo, A., Della Corte, A., Greco, L., Neff, P.: Wave propagation in pantographic 2D lattices with internal discontinuities (2014). arXiv: 1412.3926

49. Bersani, A.M., Della Corte, A., Greco, L., Neff, P.: An explicit solution for the dynamics of a taut string of finite length carrying a traveling mass: the subsonic case. Zeitschrift für angewandte Mathematik und Physik 67(4), 108 (2016)

50. Placidi, L., dell'Isola, F., Ianiro, N., Sciarra, G.: Variational formulation of pre-stressed solidfluid mixture theory, with an application to wave phenomena. Eur. J. Mech.-A/Solids 27(4), 582-606 (2008)

51. Madeo, A., Barbagallo, G., d'Agostino, M., Placidi, L., Neff, P.: First evidence of non-locality in real band-gap metamaterials: determining parameters in the relaxed micromorphic model. In: Proceedings of the Royal Society A, vol. 472, p. 20160169. The Royal Society (2016)

52. Madeo, A., Neff, P., Ghiba, I., Placidi, L., Rosi, G.: Band gaps in the relaxed linear micromorphic continuum (2014). arXiv:1405.3493

53. Giorgio, I.: Numerical identification procedure between a micro-cauchy model and a macrosecond gradient model for planar pantographic structures. Zeitschrift für angewandte Mathematik und Physik 67(4)(95) (2016)

54. dell'Isola, F., Della Corte, A., Giorgio, I., Scerrato, D.: Pantographic 2D sheets: discussion of some numerical investigations and potential applications. Int. J. Non-Linear Mech. 80, 200 208 (2016)

55. dell'Isola, F., Giorgio, I., Pawlikowski, M., Rizzi, N.: Large deformations of planar extensible beams and pantographic lattices: heuristic homogenization, experimental and numerical examples of equilibrium. In: Proceedings of the Royal Society A, vol. 472, p. 20150790. The Royal Society (2016)

56. Scerrato, D., Giorgio, I., Rizzi, N.: Three-dimensional instabilities of pantographic sheets with parabolic lattices: numerical investigations. Zeitschrift für angewandte Mathematik und Physik 67(3), 1-19 (2016)

57. Giorgio, I., Della Corte, A., dell'Isola, F., Steigmann, D.: Buckling modes in pantographic lattices. Comptes rendus Mecanique (2016)

58. Rahali, Y., Giorgio, I., Ganghoffer, J.F., Dell'Isola, F.: Homogenization à la piola produces second gradient continuum models for linear pantographic lattices. Int. J. Eng. Sci. 97, 148 $172(2015)$

59. Alibert, J., Della, A.: Corte. Second-gradient continua as homogenized limit of pantographic microstructured plates: a rigorous proof. Zeitschrift für angewandte Mathematik und Physik 66(5), 2855-2870 (2015)

60. Eremeyev, V.A., dell'Isola, F., Boutin, C., Steigmann, D.: Linear Pantographic Sheets: Existence and Uniqueness of Weak Solutions (2017)

61. Placidi, L., Barchiesi, E., Turco, E., Rizzi, N.L.: A review on 2D models for the description of pantographic fabrics. Zeitschrift für angewandte Mathematik und Physik, 67(5)(121) (2016)

62. Barchiesi, E., Placidi, L.: A review on models for the 3D statics and 2D dynamics of pantographic fabrics. In: Wave Dynamics and Composite Mechanics for Microstructured Materials and Metamaterials, pp. 239-258. Springer (2017) 
63. Turco, E., dell'Isola, F., Rizzi, N.L., Grygoruk, R., Müller, W.H., Liebold, C.: Fiber rupture in sheared planar pantographic sheets: numerical and experimental evidence. Mech. Res. Commun. 76, 86-90 (2016)

64. Spagnuolo, M., Barcz, K., Pfaff, A., dell'Isola, F., Franciosi, P.: Qualitative pivot damage analysis in aluminum printed pantographic sheets: numerics and experiments. Mech. Res. Commun. (2017)

65. Battista, A., Rosa, L., dell'Erba, R., Greco, L.: Numerical investigation of a particle system compared with first and second gradient continua: Deformation and fracture phenomena. Math. Mech. Solids (2016). https://doi.org/10.1177/1081286516657889

66. Greco, L., Giorgio, I., Battista, A.: In plane shear and bending for first gradient inextensible pantographic sheets: numerical study of deformed shapes and global constraint reactions. Math. Mech. Solids (2016). https://doi.org/10.1177/1081286516651324

67. Battista, A., Cardillo, C., Del Vescovo, D., Rizzi, N.L., Turco, E.: Frequency shifts induced by large deformations in planar pantographic continua. Nanomechanics Sci. Technol. Int. J. 6(2) (2015)

68. Turco, E., Golaszewski, M., Cazzani, A., Rizzi, N.L.: Large deformations induced in planar pantographic sheets by loads applied on fibers: experimental validation of a discrete lagrangian model. Mech. Res. Commun. 76, 51-56 (2016)

69. Turco, E., Barcz, K., Pawlikowski, M., Rizzi, N.L.: Non-standard coupled extensional and bending bias tests for planar pantographic lattices. Part i: numerical simulations. Zeitschrift für angewandte Mathematik und Physik 67(5), 122 (2016)

70. Turco, E., Rizzi, N.L.: Pantographic structures presenting statistically distributed defects: numerical investigations of the effects on deformation fields. Mech. Res. Commun. 77, 6569 (2016)

71. Turco, E., dell'Isola, F., Cazzani, A., Rizzi, N.L.: Hencky-type discrete model for pantographic structures: numerical comparison with second gradient continuum models. Zeitschrift für angewandte Mathematik und Physik 67 (2016)

72. dell'Isola, F., Lekszycki, T., Pawlikowski, M., Grygoruk, R., Greco, L.: Designing a light fabric metamaterial being highly macroscopically tough under directional extension: first experimental evidence. Zeitschrift für angewandte Mathematik und Physik 66, 3473-3498 (2015)

73. Ganzosch, G., dell'Isola, F., Turco, E., Lekszycki, T., Müller, W.H.: Shearing tests applied to pantographic structures. Acta Polytechnica CTU Proceedings 7, 1-6 (2016)

74. Alibert, J.-J., Della Corte, A., Giorgio, I., Battista, A.: Extensional elastica in large deformation as \gamma-limit of a discrete 1D mechanical system. Zeitschrift für angewandte Mathematik und Physik 68(2), 42 (2017)

75. Dell'Isola, F., Andreaus, U., Placidi, L.: At the origins and in the vanguard of peridynamics, non-local and higher-gradient continuum mechanics: an underestimated and still topical contribution of gabrio piola. Math. Mech. Solids 20(8), 887-928 (2015)

76. De Masi, A., Galves, A., Löcherbach, E., Presutti, E.: Hydrodynamic limit for interacting neurons. J. Stat. Phys. 158(4), 866-902 (2015)

77. De Masi, A., Olla, S.: Quasi-static hydrodynamic limits. J. Stat. Phys. 161(5), 1037-1058 (2015)

78. Carinci, G., De Masi, A., Presutti, E.: Super-hydrodynamic limit in interacting particle systems. J. Stat. Phys. 155(5), 867-887 (2014)

79. Carinci, G., De Masi, A., Giardinà, C., Presutti, Errico: Hydrodynamic limit in a particle system with topological interactions. Arabian J. Math. 3(4), 381-417 (2014)

80. Chatzigeorgiou, G., Javili, A., Steinmann, P.: Unified magnetomechanical homogenization framework with application to magnetorheological elastomers. Math. Mech. Solids 19(2), 193 $211(2014)$

81. Saeb, S., Steinmann, P., Javili, A.: Aspects of computational homogenization at finite deformations: a unifying review from reuss' to voigt's bound. Appl. Mech. Rev. 68(5), 050801 (2016)

82. Javili, A., Chatzigeorgiou, G., Steinmann, P.: Computational homogenization in magnetomechanics. Int. J. Solids Struct. 50(25), 4197-4216 (2013) 
83. Cazzani, A., Malagù, M., Turco, E.: Isogeometric analysis: a powerful numerical tool for the elastic analysis of historical masonry arches. Contin. Mech. Thermodyn. 28(1-2), 139-156 (2016)

84. Cazzani, A., Stochino, F., Turco, E.: An analytical assessment of finite element and isogeometric analysis of the whole spectrum of Timoshenko beams. ZAMM-Journal of Applied Mathematics and Mechanics/Zeitschrift für Angewandte Mathematik und Mechanik (2016)

85. Cazzani, A., Stochino, F., Turco, E.: On the whole spectrum of Timoshenko beams. Part I: a theoretical revisitation. Zeitschrift für angewandte Mathematik und Physik 67(2), 1-30 (2016)

86. Cazzani, A., Malagù, M., Turco, E., Stochino, F.: Constitutive models for strongly curved beams in the frame of isogeometric analysis. Math. Mech. Solids 21(2), 182-209 (2016)

87. Greco, L., Cuomo, M.: An isogeometric implicit G1 mixed finite element for Kirchhoff space rods. Comput. Methods Appl. Mech. Eng. 298, 325-349 (2016)

88. Cuomo, M., Contrafatto, L., Greco, L.: A variational model based on isogeometric interpolation for the analysis of cracked bodies. Int. J. Eng. Sci. 80, 173-188 (2014)

89. Greco, L., Cuomo, M.: B-spline interpolation of kirchhoff-love space rods. Comput. Methods Appl. Mech. Eng. 256, 251-269 (2013)

90. Greco, L., Cuomo, M.: An implicit G1 multi patch B-spline interpolation for kirchhoff-love space rod. Comput. Methods Appl. Mech. Eng. 269, 173-197 (2014)

91. Greco, L., Cuomo, M.: Consistent tangent operator for an exact kirchhoff rod model. Contin. Mech. Thermodyn. 27(4-5), 861-877 (2015) 